medRxiv preprint doi: https://doi.org/10.1101/2020.03.27.20045237.this version posted May 7, 2020. The copyright holder for this preprint (which was not certified by peer review) is the author/funder, who has granted medRxiv a license to display the preprint in perpetuity. All rights reserved. No reuse allowed without permission.

\title{
1 STeCC: Smart Testing with Contact Counting Enhances Covid-19 Mitigation by Bluetooth 2 App Based Contact Tracing
}

\author{
Hossein Gorji ${ }^{2 *}$, Markus Arnoldini ${ }^{2 *}$, David F. Jenny ${ }^{3}$, Wolf-Dietrich Hardt ${ }^{4 \#}$, Patrick Jenny ${ }^{5 \#}$
}

${ }^{1}$ École Polytechnique Fédérale de Lausanne, MCSS, CH-1015 Lausanne, Switzerland

${ }^{2}$ Department of Health Sciences and Technology, ETH Zurich, CH-8093 Zurich, Switzerland

${ }^{3}$ Fliweg 19, 8872 Weesen, Switzerland

${ }^{4}$ Institute of Microbiology, D-BIOL, ETH Zurich, CH-8093 Zurich, Switzerland

${ }^{5}$ Department of Mechanical and Process Engineering, ETH Zurich, IFD, CH-8092 Zurich, Switzerland

*contributed equally

\#contributed equally

Correspondence: jenny@ifd.mavt.ethz.ch

\section{Abstract}

SARS-CoV2 spread is hard to control, as asymptomatic people contribute to transmission. Currently, Covid-19 mitigation imposes social distancing and isolates the diseased. This slows down virus spread, eases stress on health care systems and thereby reduces the death toll. However, this strategy takes a high economic toll, and virus transmission will surge again if measures are lifted. App-based contact tracing of symptomatic cases and isolating their contacts has been proposed as an alternative, but may not suffice for mitigation, as asymptomatic infections remain unidentified. Here, we evaluate complementary mitigation strategies relying on virus-RNA testing to detect and quarantine both, symptomatic and asymptomatic cases. Epidemic dynamics modeling shows that stopping the pandemic by mass testing alone is unrealistic, as we lack enough tests. However, realistic numbers of tests may suffice in a smart-testing strategy, e.g. when biasing tests towards people with exceptionally high numbers of contacts. These people are at particularly high risk to become infected (with or without symptoms) and transmit the virus. A mitigation strategy combining smart testing with contact counting (STeCC) and contact tracing in one app would reduce $R_{0}$ by 2.4 -fold (e.g. from $R_{0}=2.4$ to $R_{0}=1$ ) with realistic test numbers ( $\approx 166$ per 100 '000 people per day) when a realistic fraction of smartphone owners use the app ( $\approx 72 \%$, i.e. $\approx 50 \%$ in total population). Thereby, STeCC expands the portfolio of mitigation strategies and may help easing social distancing without compromising public health.

\section{Main}

The Covid-19 pandemic has evaded initial containment measures. Current responses have therefore shifted towards mitigating the effects. However, proven vaccines and therapies are lacking and the current capacity for detecting the virus via its genomic RNA is limited (1). Thus, mitigation in many countries relies on extreme social distancing and diagnosing virus (SARS-CoV2) infections in infected people showing mild to severe symptoms. In combination, these two measures slow down pandemic spread and avoid overburdening healthcare systems, thus easing the demand for intensive care. However, this strategy has three major shortcomings. First, the limitation of testing to diseased people handicaps the efficiency assessment of current mitigation measures (i.e., reduce the basic virus reproduction number 
medRxiv preprint doi: https://doi.org/10.1101/2020.03.27.20045237.this version posted May 7, 2020. The copyright holder for this preprint (which was not certified by peer review) is the author/funder, who has granted medRxiv a license to display the preprint in perpetuity. All rights reserved. No reuse allowed without permission.

$\left.R_{0}<1\right)$. Second, it leaves many infected people with mild or no symptoms undetected (2), and therefore renders them more likely to infect others. Third, as social distancing measures limit non-essential business, it imposes severe economic consequences, which worsen over time. A broad social-distancing-based approach is therefore not sustainable, but mitigation measures need to stay in place until effective therapies or vaccines become available to avoid a second wave of virus spread. As these options are still months away, we need to consider alternative mitigation strategies.

Smartphone apps for Bluetooth-based contact tracing, such as the European PEPP-PT project, the British NHSX, or the Swiss D3PT, can help to identify individuals that have recently had an infection-relevant contact (i.e. one that confers a risk of transmission) with known Covid-19 cases and might therefore have benn infected. This can help to mitigate the pandemic $(3,4)$. However, quantitative modeling suggests that this may not suffice to replace social distancing completely, i.e. if some of the assumptions about the disease dynamics turn out to be unrealistic. Specifically, this approach may fail if $<56 \%$ of the average population use the app, if the epidemic doubling time is $<3.5$ days, if $>35 \%$ of the infected people are asymptomatic, or if they were more than 0.3 -fold as infectious as symptomatic cases ((3); Fig. S5). Infectiousness of asymptomatic cases has been estimated to be between $10 \%$ and $100 \%$ of symptomatic cases (4-6). The asymptomatic cases are a particular problem, as app-based contact tracing detects such cases insufficiently. Therefore, we have looked for an alternative approach, based on testing for virus RNA, a marker for active infections with or without symptoms (Fig. 1, highlighted in dark or light blue).

First, we asked how well mass testing random samples of the population could complement or replace social distancing. In theory, this can be achieved by testing large fractions of the population at regular intervals, and isolating people who test positive. Using a deterministic modeling approach that explicitly considers infected cases with and without symptoms, we can estimate how many tests per 100'000 people per day would suffice to achieve the same effect as current social distancing measures which have reduced $R_{0}$ from 2.4-3 to less than 1, e.g. in Germany or Switzerland $((7,8)$; for technical details, see supplementary text, table $\mathbf{S 2}$ ). We will first present our findings and then discuss how testing could be realistically implemented, e.g. by combining it with serological testing or app-based technologies. 
medRxiv preprint doi: https://doi.org/10.1101/2020.03.27.20045237.this version posted May 7, 2020. The copyright holder for this preprint (which was not certified by peer review) is the author/funder, who has granted medRxiv a license to display the preprint in perpetuity. All rights reserved. No reuse allowed without permission.

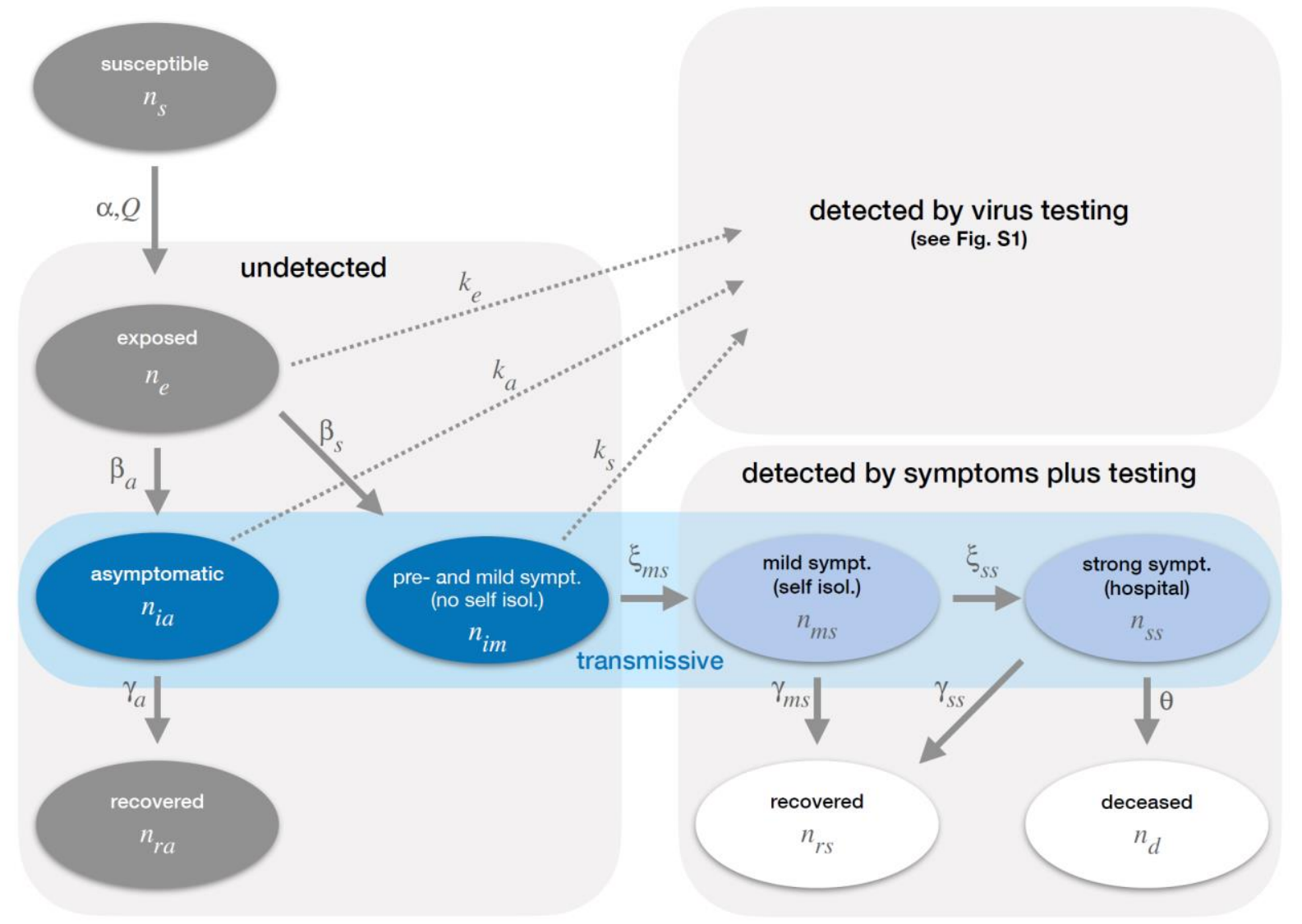

Figure 1. Graphical illustration of the modeling approach showing the dependencies within the system describing the dynamics of the susceptible, undetected and detected infected populations. It is crucial that the model distinguishes between individuals detected by symptoms (with or without testing; light blue), and those detected by virus testing (dark blue).

To estimate how mitigation strategies affect $R_{0}$, we use a mathematical model that employs a set of ordinary differential equations to describe the dynamics of the infection of a susceptible population (Fig. 1, supplementary text section 1, Fig. S1, table S1). To obtain realistic simulations, we parametrize our model using published data ((5, 9-14); table S2; supplementary text section 2 ). Infection of susceptible people will lead to a latency phase (Fig. 1, exposed). The exposed will later become transmissive, and either remain asymptomatic and recover or become pre-symptomatic and later develop mild symptoms (Fig. 1, dark blue; see parameters in table S2). These two transmissive groups do not know that they are infected, and remain unidentified in current mitigation approaches. Some people with mild symptoms of disease will self-isolate (Fig. 1, light blue). Due to self-isolation, they will have a reduced probability to infect others (we assume a 90\% reduction). Infected people with severe symptoms are hospitalized, immediately isolated under strict quarantine and do not infect others (Fig. 1, light blue). The same applies for anyone else who is tested virus-positive. In addition, we consider the effect of overloading the health system. After all intensive care units (ICUs) are occupied, additional cases requiring intensive care will suffer an elevated death rate (Fig. 1; supplementary text, section 2). As our model consists of a set of ordinary differential equations, which become linear in the early stages of the pandemic (when nearly the whole population still is susceptible), we can now analytically test how particular mitigation strategies affect $\mathrm{R}_{0}$ (supplementary text, sections 3,4 and 5 ). 
medRxiv preprint doi: https://doi.org/10.1101/2020.03.27.20045237.this version posted May 7, 2020. The copyright holder for this preprint (which was not certified by peer review) is the author/funder, who has granted medRxiv a license to display the preprint in perpetuity. All rights reserved. No reuse allowed without permission.

A
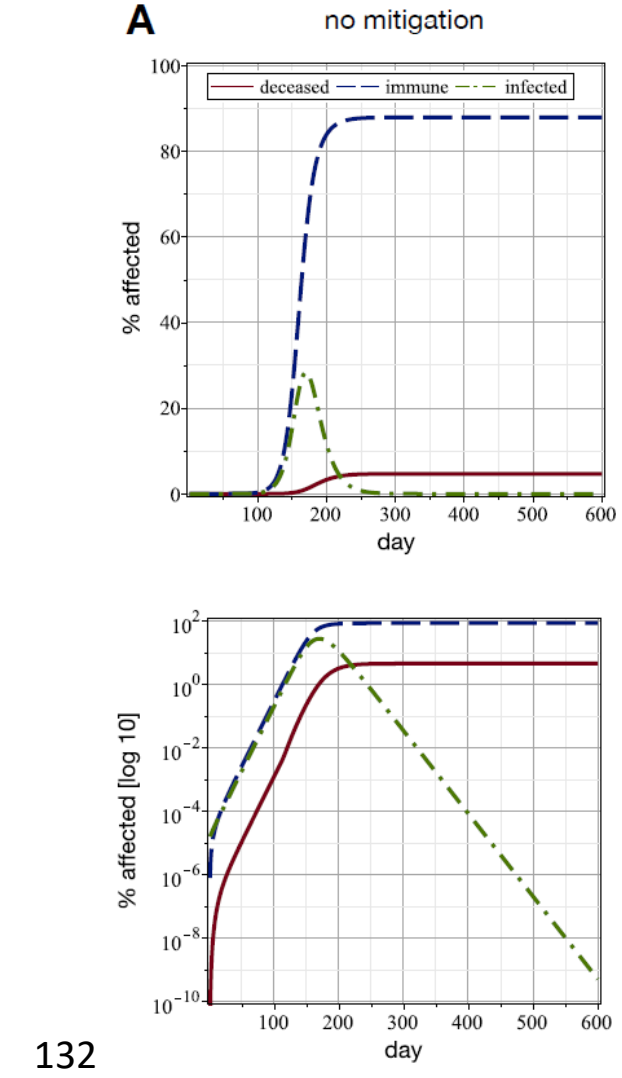

B
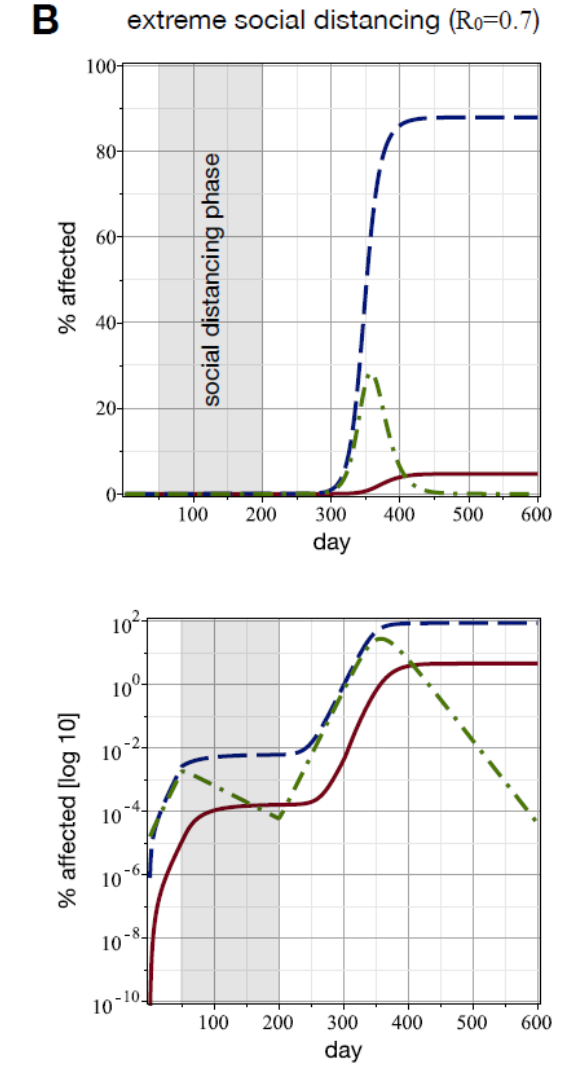
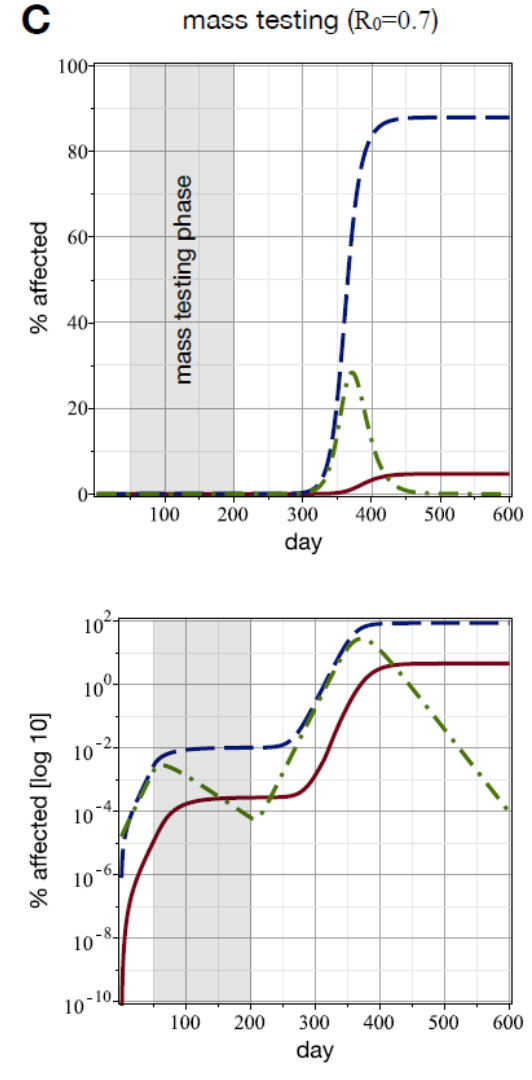

In order to compare different mitigation strategies to a baseline, we first describe what the model predicts if we omit any mitigation. Here, we assume a value for $R_{0}$ that is in the mid-range of published estimates, i.e. that one infected person infects 2.4 others in average ( $R_{0}=2.4$; Fig. 2A; alternative $R_{0}$ values are tested in Fig. S2A,B). Under these circumstances, $87 \%$ will either recover or die from the disease within $\approx 250$ days, which compares well to the $81 \%$ predicted by (5) for UK and US populations in the absence of mitigation plans. We also predict more than $4 \%$ of the population to be killed by the virus, and ICUs to be at maximum capacity for $\approx 150$ days of that year. For the unmitigated case these numbers are going to change only by 2 -fold or less, if alternative plausible values are assumed for $\mathrm{R}_{0}$ (1.9 or 2.9; Fig. S2A,B) or for the availability of ICU beds (Fig. S2C). Thus, our basic predictions are robust, even if input parameters for the virus infection dynamics (table S2) might be subject to change when more precise parameters became available. Please note that Fig. 2A depicts the worst-case scenario, which can be improved by mitigation.

We then compare the effects of extreme social distancing with those of extensive mass testing. Extreme social distancing is imposed for a period of 150 days (day 50-200 of the pandemic), reducing $R_{0}$ to 0.7 (table $S 1$ ). This is achieved if the rate of infection of susceptible people is reduced by $71 \%$ (Fig. 2B; data for $R_{0}=1$, as recently achieved in Switzerland (8), is shown in Fig. S4A). In line with current observations, extreme social distancing dramatically decreases the fraction of people experiencing an infection and reduces the number of deaths $(<0.0001 \%$ in Fig. 2B), at least if social distancing is initiated early on (e.g. by day 50) and strictly adhered to for months. The fraction of the infected population declines during extreme social distancing. This is in line with other modeling studies (3). However, once these measures are abandoned, the infection starts spreading again, leading to a similar death toll and ICU overloading as in the case without mitigation (Fig. 2B, days 200-600). 
medRxiv preprint doi: https://doi.org/10.1101/2020.03.27.20045237.this version posted May 7, 2020. The copyright holder for this preprint (which was not certified by peer review) is the author/funder, who has granted medRxiv a license to display the preprint in perpetuity. All rights reserved. No reuse allowed without permission.

Figure 2. Social distancing and extensive mass testing alone have qualitatively equal mitigating effects. We used the model described in the supplementary text. (A) Model outcome if no mitigation strategies are in place $\left(R_{0}=2.4\right.$; see table S3 for additional input parameters). (B) Model outcome if extreme social distancing $(71 \%$ lower infection rate, leading to $\mathrm{R}_{0}=0.7$; table S2) is in place between days 50 and 200 of the outbreak. After day 200, social distancing is discontinued. (C) Model outcome if mass testing with isolation of detected cases is applied between days 50 and 200 of the outbreak (as shown in A). The shown effect is achieved if $37^{\prime} 300$ people per $100^{\prime} 000$ are tested every day ( $5 \%$ false negative rate; test speed $=1$ day). Tables S2 and S3 show all other model parameters. Testing is discontinued after day 200. Dashed blue lines represent recovered plus infected plus deceased, dashed-dotted green lines infected, and solid red lines deceased people. Lower panels show the same data as upper panels, but with a $\log _{10}$-scale for $Y$-axes.

Next, we analyze a mitigation strategy that is based on mass testing alone (supplementary text section 3). We would test random samples of the population with concomitant isolation of detected cases. The test is assumed to take one day to process, to yield $5 \%$ false negative results (discussed below), which is technically reasonable, and to result in immediate quarantine of the virus-positive cases. Figure S3A shows the factor by which $\mathrm{R}_{0}$ changes as a function of number of tests and for different test processing times; the same is shown in Fig. S3B for a higher false negative rate. Again, we assume that the mitigation strategy is implemented during days $50-200$ of the pandemic. Applying 37'300 tests per 100 '000 people per day yields the same outcome as extreme social distancing $\left(R_{0}=0.7\right.$; Fig. 2C, compare to Fig. 2B; supplementary text section 3 for details). Reducing the mass testing to $12^{\prime} 600$ tests per $100^{\prime} 000$ people per day is sufficient to keep the number of infections constant $\left(R_{0}=1\right.$, Fig. S4B $)$ and yields equivalent results as a moderate form of social distancing (Fig. S4A). Both interventions can dramatically slow the epidemiological dynamics, but similarly to the previously discussed social distancing, the number of infections and deaths will start to rise again after the testing regime is abandoned (Fig. 2C, days 200-600).

It is easy to see that both, mass testing and social distancing, can have qualitatively identical effects on the epidemiological dynamic, but they work differently. Social distancing decreases the overall infection rate by reducing the freedom of movement for all, whereas mass testing will allow us to limit the isolation to the infected fraction of the population that would transmit the virus. The latter would have the important advantage of inflicting much smaller burdens on the economy than social distancing, as far smaller fractions of the population need to be isolated. However, the number of tests we predict to be necessary to reach $R_{0} \leq 1$ (12'600 tests per 100'000 people per day; Fig. S4B) is clearly unrealistic for now, and $>50$-fold above the current testing capacities even of countries with highly developed and well-funded healthcare systems. Optimizing the test specificity or the test speed changes the required number of tests by merely $<2$-fold (Fig. S3). Thus, mass testing alone is not an option.

We therefore analyzed mitigation strategies combining virus testing with other measures. This should reduce the number of tests needed to achieve $R_{0} \leq 1$. First, we tested a mitigation strategy combining mild social distancing, which reduces the infection rate by $33 \%$ (this would yield $R_{0}=1.6$, if applied alone; table S1), and mass testing (assumed test speed = 1 day, $5 \%$ false negatives). This may approximate a scenario where mass testing is applied to balance the effects of easing current social distancing measures. Using this combined strategy, we would require 4'500 tests per 100 '000 people per day to reach $R_{0}=1$ (Fig. 3A). However, this still exceeds the current test capacities.

An additional mitigation measure is serological testing to identify the subpopulation that has already recovered from the disease and is now immune (15). This could be combined with mass testing to reduce the number of tests by removing the immune subpopulation from the pool of candidates to be tested. However, we are still at a stage of this pandemic where 
medRxiv preprint doi: https://doi.org/10.1101/2020.03.27.20045237.this version posted May 7, 2020. The copyright holder for this preprint (which was not certified by peer review) is the author/funder, who has granted medRxiv a license to display the preprint in perpetuity. All rights reserved. No reuse allowed without permission.

the fraction of immune people is likely quite small (see Fig. 2A). Therefore, serological testing could only minimally reduce the number of needed tests per 100'000 people per day. In no way are we implying, however, that serological testing is not useful: especially in the case of healthcare workers, and other essential personnel that is in close contact with the public, it is of great value to know who is immune to the disease. Also, serological testing could complement mitigation at later phases of such an epidemic when larger fractions of the population have become immune (Fig. 2A; $>10 \%$ immune or deceased beyond day 140).
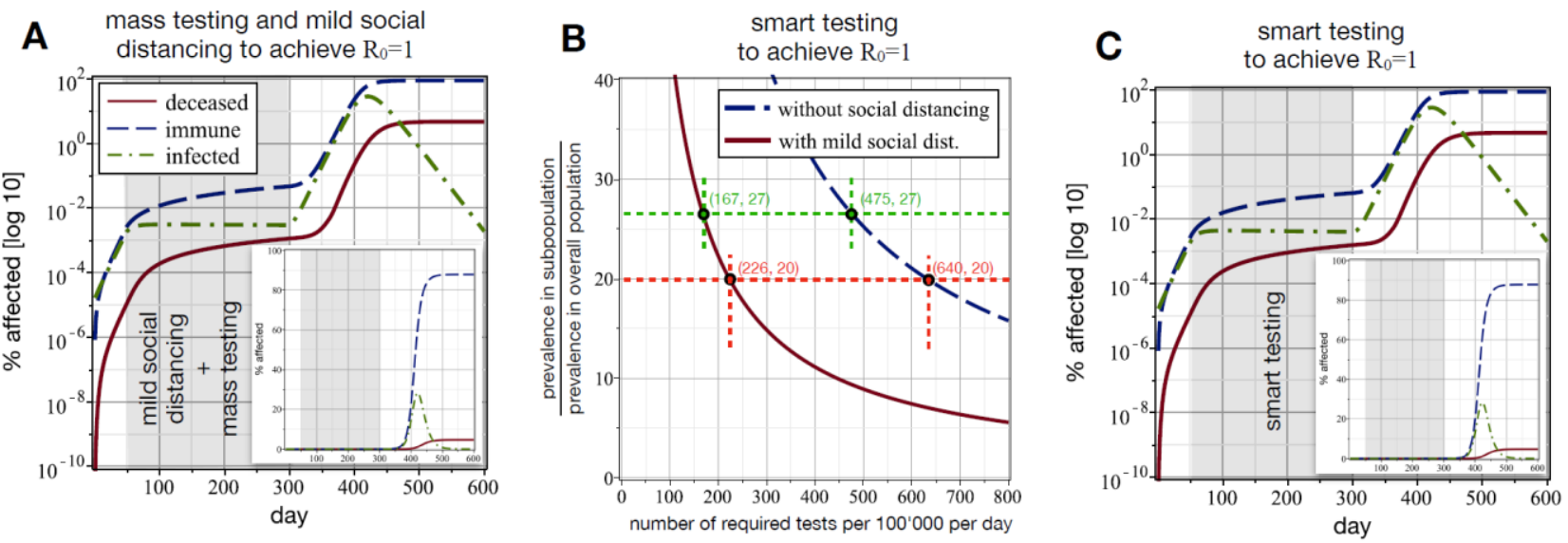

Figure 3. Effective combinations of mass testing with other mitigation strategies can be achieved with realistic numbers of tests. (A) A combination of mild social distancing ( $33 \%$ reduction in infection rate; $R_{0}=1.6$ if it were applied alone) and mass testing (4'500 tests per 100'000 per day; false negative rate = 5\%; test speed = 1 day) during days 50-300 can reduce Ro to 1 . (B) Smart selection of the tested subpopulation reduces the required test numbers, if additional information allows identifying a subpopulation with higher than average infection prevalence (details, below). The Y-axis shows the factor by which the infection prevalence increases in the tested subpopulation. This will reduce the required number of tests. Blue dashed line: smart testing alone is used to achieve $R_{0}=1$ (if $R_{0}=2.4$ for the case without mitigation); red solid line: smart testing combined with mild social distancing (33\% reduction in infection rate by social distancing). Green and red points illustrate examples discussed, below. (C) The same effect as in A can be achieved with smart testing, assuming that we can find a subpopulation with a 27 -fold increased infection prevalence. Here, we need 475 tests per 100 '000 per day to reach $R_{0}=1$ (green dots in panel $B$ ). $(A, C)$ : Dashed blue lines: recovered plus infected plus deceased; dasheddotted green lines: infected; solid red lines: deceased people. Inserts show the same data, but with a linear scale for $Y$-axes. All presented cases assume false negative rate $=5 \%$; test speed $=1$ day.

Finally, we assessed how virus testing could be combined with Bluetooth-based tracing applications (e.g. PEPP-PT (16)), which are designed to identify individuals who had recent contacts with infected people and might therefore be infected, too. Contact tracing alerts the detected contacts to encourage isolation or virus testing. However, as this strategy misses most transmission events by asymptomatically infected cases and cases with mild symptoms that remained undetected (Fig. 1, dark blue), it is unlikely to suffice alone, given the epidemiological parameters in Europe (3) (Fig. S5, supplementary text section 4). Therefore, we explored an alternative strategy that relies on smart testing. In theory, smart testing would use additional information to focus virus tests on subpopulations having a higher prevalence than the overall population. We will first analyze the theoretical principle and then discuss a practical implementation.

For smart testing to work, two crucial requirements have to be fulfilled. The prevalence in the tested subpopulation has to be high enough, so that a limited number of tests will suffice to detect the necessary number of infected people. Furthermore, the traced 
medRxiv preprint doi: https://doi.org/10.1101/2020.03.27.20045237.this version posted May 7, 2020. The copyright holder for this preprint (which was not certified by peer review) is the author/funder, who has granted medRxiv a license to display the preprint in perpetuity. All rights reserved. No reuse allowed without permission.

subpopulation featuring the desired prevalence has to be large enough. We describe the detailed derivation of the corresponding mathematical expressions in the supplementary text section 5 . Smart testing alone can substantially reduce the number of virus tests needed to achieve $\mathrm{R}_{0}=1$ (Fig. 3B, dashed blue line). In fact, if we assume that smart testing identifies a subpopulation with 27 -fold higher prevalence than the overall population, we can achieve $\mathrm{R}_{0}=1$ with 475 tests per 100'000 per day (Fig. 3B, dashed blue line, green dot) and thus keep the number of infections constant as long as smart testing is maintained (Fig. $\mathbf{3 C}$, days 50 300). Fewer tests would be needed, if we could further increase the virus prevalence in the tested subpopulation. Alternatively, one could combine smart testing with mild social distancing, e.g. to stop a surge of incidence when strong distancing measures are eased. For example, if social distancing would reduce the infection rate by merely $33 \%$, we would only need 167 tests per $100^{\prime} 000$ per day to reach $R_{0}=1$ (Fig. 3B, red line, green dot). Sufficient testing capacity for this combined strategy would already be available today in several European countries. We conclude that smart testing could work: it could achieve the same result as mass testing (Fig. S4B), but with much fewer tests (Fig. 4B).

Can we use Bluetooth-based tracing apps for identifying high prevalence subpopulations? A first attempt at achieving this would be an adaptation of app-based contact tracing to identify individuals with recent infection-relevant contact to detected Covid-19 cases (4). This contact subpopulation should have a higher prevalence than the overall population. However, such a "trace+test" approach may not suffice to achieve $R_{0}=1$, at least when applied without any other mitigation strategies (3). This is explained by its focus on contacts of symptomatic individuals (while contacts to asymptomatic cases are missed), as well as the virus' infection and transmission dynamics (for details, see the supplementary text section 6). Contact tracing is therefore not sufficient to reach the requirements for smart testing to work as a standalone mitigation strategy.

As many asymptomatic SARS-CoV2 carriers contribute to the transmission, we reasoned that smart testing should cover symptomatic and asymptomatic cases, alike. To solve this problem, we suggest a different strategy for identifying high-prevalence subpopulations. Importantly, this can be achieved using the same Bluetooth based technology as contact tracing. Our strategy relies on the fact that some individuals will have many more infection-relevant contacts than most others (17). For example, with realistic scale-free network model assumptions, $\approx 1 \%$ of the population with the most infectionrelevant contacts (at least 10 times more than the average) make the difference between $R_{0}=1$ and $R_{0}=2.4$ (see supplementary text section 6 ). These individuals can be identified by counting of infection-relevant contacts (i.e. contacts which could facilitate transmission), regardless, if contacts were infected or not. Their sheer number of contacts makes highcontact individuals much more likely than others to become infected and to transmit the virus, and they are known to be highly important for epidemiological dynamics ((18), referred to as super-spreaders). To test if smart testing with contact counting (STeCC; Fig. 4A) works in realistic scenarios, we used a scale free network model, which can account for the heterogeneity of the number of contacts in a population (supplementary text section 6). The fraction of app-users in the population will also affect the success of STeCC. We assume that children under age 10 contribute little to transmission in the general population $(19,20)$ and would not use smartphones, as suggested by others (3). High-risk persons (i.e. $>70$ years old; $20 \%$ of the overall population) would remain shielded in isolation for safety reasons and would therefore not contribute to the infection dynamics. People aged 10-70 (in particular 
medRxiv preprint doi: https://doi.org/10.1101/2020.03.27.20045237.this version posted May 7, 2020. The copyright holder for this preprint (which was not certified by peer review) is the author/funder, who has granted medRxiv a license to display the preprint in perpetuity. All rights reserved. No reuse allowed without permission.

the high-contact subgroup) would use smartphones, and we assessed how their app-usage would affect mitigation.

Then, we tested three different scenarios how STeCC could be implemented to reach $R_{0}=1$. We used a progressive testing cycle, as testing the same high-contact individuals every day would seem unrealistic. On the first day, based on the number of available tests, we would identify the optimal subgroup with the highest frequency of infection-relevant contacts, and invite them to be tested (Fig. 4B; dark blue; supplementary text section 6). During the next days, we would repeat the same procedure, but exclude the groups previously identified. After 7 days, we would begin the cycle again and identify the optimal high-contact group from the entire susceptible population. Using this testing cycle, we observed that STeCC alone could reduce $R_{0}$, but cannot reach $R_{0}=1$ with realistic numbers of app-users and tests per day (Fig. S6). Next, we assessed a STeCC strategy, which would refer two groups of people to quarantine, i.e. the high-contact individuals that tested positive and their recent contacts. This strategy would reach $\mathrm{R}_{0}=1$, if $90 \%$ of smartphone users would use the app (63\% of the overall population) and $\approx 397$ tests per 100'000 people per day were carried out (Fig. 4C, red lines). Finally, we tested a combination of the latter STeCC approach with Bluetooth app-based contact tracing (similar to (4)), which may be possible using the same data. This would refer three groups of people to quarantine, i.e. the high-contact individuals testing positive, their recent contacts and the contacts of symptomatic individuals. If $72 \%$ of smartphone users would use the app $(\approx 50 \%$ of the overall population) we would need $\approx 166$ tests per $100^{\prime} 000$ people per day to reach $R_{0}=1$ (Fig. 4D, red lines). This testing capacity is currently available in several European countries, like Switzerland. Please note that our example in Fig. 4D assumes an $R_{0}=2.4$, if no mitigation was applied. An identical 2.4-fold reduction is achievable for any other basic virus reproduction rate, as long as $72 \%$ of smartphone users would use the app and $\approx 166$ tests per 100'000 people per were carried out. We have conducted sensitivity studies to study effects of the false negative rate, network parameters, test processing times and $\mathrm{R}_{0}$ for the unmitigated case. In all considered variations, STeCC with contact tracing could achieve $R_{0}=1$ with reasonable fractions of appusers and test numbers (see supplementary text section 6). Thus, STeCC with contact tracing could add to any mitigation policy. 
A
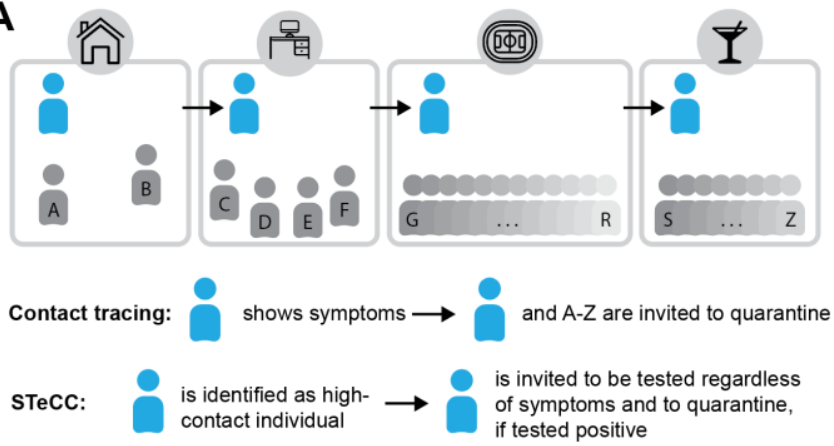

STeCC

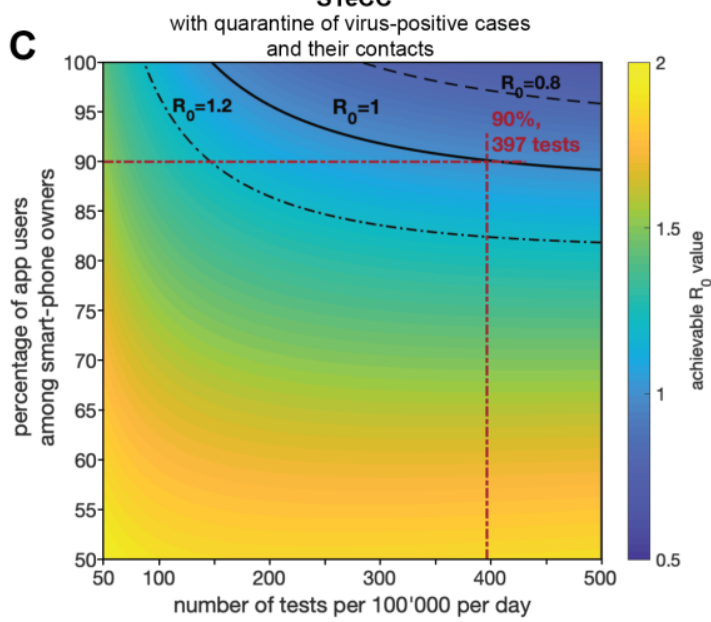

B

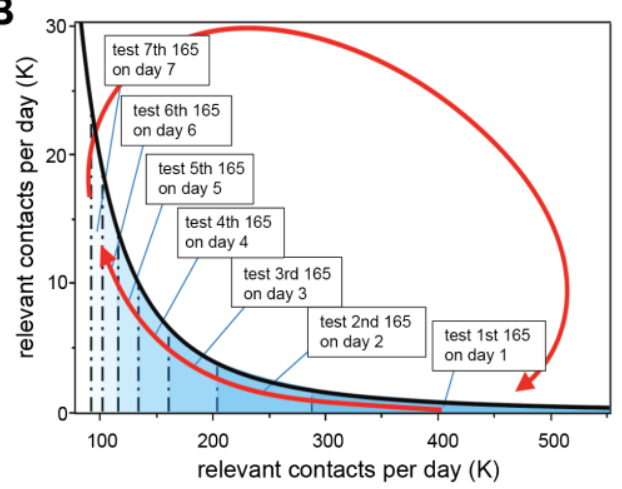

STeCC

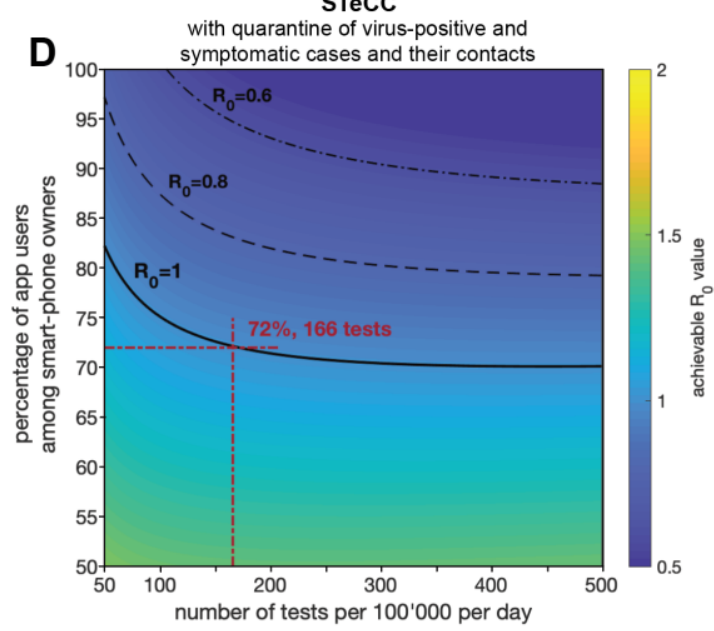

Figure 4. STeCC can achieve $\mathbf{R}_{0}=1$ using a realistic number of tests and realistic rates of app-usage. (A) STeCC

approach vs contact tracing. Contacts of a focal individual are logged using software. Using contact tracing, all contacts would be quarantined if the focal individual shows symptoms. Using STeCC, the focal individual is tested for infection if their contacts exceed a threshold number (e.g., if they visit a sporting event and a bar, in addition to being at home and at work), and quarantined if tested positive. (B) Progressive test cycling approach. Example for $80 \%$ app users among smart-phone owners and the availability of 165 tests per 100 '000 people per day. (C) Covid-19 mitigation by STeCC alone with quarantine of contacts of virus-positive cases. Graph showing how the number of tests per 100'000 per day relates to the percentage of app users. We assume $R_{0}=2.4$ for the unmitigated case, test speed of 1 day, immediate notification and quarantine, false negative ratio $5 \%$, asymptomatic cases ( $1 / 3$ of all) are $50 \%$ as infective as symptomatic cases. Solid line: values to achieve $R_{0}=1$; dashed line: values to achieve $R_{0}=0.8$; dashed dotted line: values to achieve $R_{0}=1.2$. (D) Covid-19 mitigation by combining STeCC with app-based contact tracing. Graph showing how the number of tests per 100'000 per day relates to the percentage of app users. We assume $R_{0}=2.4$ for the unmitigated case, test speed of 1 day, immediate notification and quarantine, false negative ratio $5 \%$, asymptomatic $(1 / 3$ of all) are $50 \%$ as infective as symptomatic cases. Solid line: values to achieve $R_{0}=1$; dashed line: values to achieve $R_{0}=0.8$; dashed dotted line: values to achieve $R_{0}=0.6$. The coloring indicates the achievable $R_{0}$ with the respective mitigation strategy in place (see supplementary text, sections 6 and 7).

What are the advantages of a STeCC-based mitigation strategy? First, it includes detection and removal of asymptomatic cases and their contacts. In contrast to contact tracing alone (Fig. S6), the combination with STeCC would work when $>72 \%$ of the smartphone users $(\approx 50 \%$ of the overall population) would use the app, or if unfavorable assumptions about the pandemic's parameters would turn out to be true (Fig. S8). This synergy is attributable to the different selection processes of both approaches. Furthermore, STeCC could be implemented within the same apps as developed for contact tracing. This could offer additional choices for app-based mitigation, i.e. the parallel use of STeCC and 
medRxiv preprint doi: https://doi.org/10.1101/2020.03.27.20045237.this version posted May 7, 2020. The copyright holder for this preprint (which was not certified by peer review) is the author/funder, who has granted medRxiv a license to display the preprint in perpetuity. All rights reserved. No reuse allowed without permission.

contact tracing, which is particularly powerful (Fig. 4D). As STeCC focusses on a small highcontact group, it works with relatively small numbers of tests and would only quarantine those that are virus positive (and their contacts, e.g. Fig. 4C,D). It is realistic given the available test capacities in several countries and would affect smaller fractions of the population than other mitigation strategies (i.e. contact tracing or social distancing). Thereby, one could enhance the impact of Bluetooth-based tracing applications. Due to its key function in virus transmission, the identified high-contact subgroup would also be a promising priority for vaccination, once limited supplies of a vaccine become available.

What are the limitations of STeCC? First, we cannot exclude that some assumptions used in our model may be too optimistic or that more precise information might be derived later from alternative, more detailed simulation approaches. However, modeling the impact with less favorable parameters verified that STeCC would still provide substantial benefits (Fig. S7, Fig. S8). Second, contact counting has not been a focus during the development of Bluetooth-based proximity testing applications. Thus, small adaptations might be needed, in order to enable efficient detection and notification of high-contact individuals.

Strict social distancing has been successful in achieving $R_{0}<1$ in many countries, but at a high economical and societal cost. Easing of these measures is presently being discussed or implemented. However, if a large fraction of the population has remained susceptible, a second wave of disease is bound to occur in the absence of effective alternative mitigation strategies. We suggest using a combination of contact tracing and STeCC, as a simple mitigation approach which relies on identifying high-contact individuals, testing them for infection, and quarantining positive cases. STeCC requires the same information as contact tracing (a list of unique contacts in a given period of time), and can be implemented using the same information as the contact tracing apps that are currently being developed or already in use. STeCC would be achievable using a number of tests that is realistic today in several European countries, and can be achieved in many others with appropriate efforts.

STeCC adds to the portfolio of mitigation strategies for the Covid-19 pandemic. It could be deployed quickly in countries with sufficient testing capacities like Switzerland (capacity $\approx 230$ tests per $100^{\prime} 000$ per day). A combination with contact tracing might be particularly powerful. Our study provides quantitative estimates for the number of tests needed by starting out with realistic assumptions about the relevant parameters, like number of app users. Once STeCC is applied, one can adjust the strategy flexibly in order to ensure the desired performance. STeCC offers a realistic approach to help relaxing broad social distancing policies in the near future without compromising health, while at the same time providing public health officials with much needed actionable information on the success of their interventions. This will be an important prerequisite for reclaiming our normal public life and initiating economic recovery.

\section{Acknowledgements:}

The authors are very thankful to Dario Ackermann, who created the website with the simulation tool based on the model presented in this paper. The authors would like to thank Emma Slack, Erik Bakkeren and Noemi Santamaria for helpful comments on the manuscript. HG acknowledges funding from the Swiss National Science foundation (grant number 174060).

\section{Methods:}


medRxiv preprint doi: https://doi.org/10.1101/2020.03.27.20045237.this version posted May 7, 2020. The copyright holder for this preprint (which was not certified by peer review) is the author/funder, who has granted medRxiv a license to display the preprint in perpetuity. All rights reserved. No reuse allowed without permission.

410

411

412

413

414

The dynamic model was implemented with Maple 2018. The calculations for mass testing, contact tracing and smart testing were implemented with MATLAB and the Statistics Toolbox Release 2018b. They will be made available at GitHub.

\section{References:}

1. Johns Hopkins University, Worldometer (2020) (available at

https://www.worldometers.info/coronavirus/).

2. R. Li et al., Substantial undocumented infection facilitates the rapid dissemination of novel coronavirus (SARS-CoV2). Science. 6, eabb3221-9 (2020).

3. R. Hinch et al., Effective Configurations of a Digital Contact Tracing App: A report to NHSX. A report to NHSX, 1-29 (2020).

4. L. Ferretti et al., Quantifying SARS-CoV-2 transmission suggests epidemic control with digital contact tracing. Science, eabb6936-13 (2020).

5. N. Ferguson et al., Impact of non-pharmaceutical interventions (NPIs) to reduce COVID19 mortality and healthcare demand. Imperial College COVID-19 Response Team (2020), doi:10.25561/77482.

6. E. Lavezzo et al., Suppression of COVID-19 outbreak in the municipality of Vo, Italy. medRxiv, 1-23 (2020).

7. Robert Koch Institut, Täglicher Lagebericht des RKI zur Coronavirus-Krankheit-2019 (COVID-19), 1-10 (2020).

8. J. Scire et al., "Reproductive number of the COVID-19 epidemic in Switzerland with a focus on the Cantons of Basel-Stadt and Basel-Landschaft" (2020), pp. 1-13.

9. D. Baud et al., Real estimates of mortality following COVID-19 infection. Lancet Infect Dis, 1-1 (2020).

10. Y. Liu, A. A. Gayle, A. Wilder-Smith, J. Rocklöv, The reproductive number of COVID-19 is higher compared to SARS coronavirus. Journal of Travel Medicine. 27, 1-4 (2020).

11. Z. Liu, X. Bing, Z. Zhi, The epidemiological characteristics of an outbreak of 2019 novel coronavirus diseases (covid-19) in China. Novel Coronavirus Pneumonia Emergency Response Epidemiology Team. 41 (2020).

12. K. Men et al., Estimate the incubation period of coronavirus 2019 (COVID-19). medRxiv, 1-13 (2020).

13. V. Virlogeux et al., Incubation Period Duration and Severity of Clinical Disease Following Severe Acute Respiratory Syndrome Coronavirus Infection. Epidemiology. 26, 666-669 (2015).

14. F. Zhou et al., Clinical course and risk factors for mortality of adult inpatients with COVID-19 in Wuhan, China: a retrospective cohort study. Lancet. 395, 1054-1062 (2020).

15. C. Sheridan, Fast, portable tests come online to curb coronavirus pandemic. Nat Biotechnol (2020), doi:10.1038/d41587-020-00010-2.

16. Pan-European Privacy-Preserving Proximity Tracing, HOME | Pepp-Pt (2020) (available at www.pepp-pt.org).

17. L. A. Meyers, B. Pourbohloul, M. E. J. Newman, D. M. Skowronski, R. C. Brunham, Network theory and SARS: predicting outbreak diversity. Journal of Theoretical Biology. 232, 71-81 (2005).

18. M. J. Keeling, K. T. D. Eames, Networks and epidemic models. J. R. Soc. Interface. 2, 295-307 (2005). 
medRxiv preprint doi: https://doi.org/10.1101/2020.03.27.20045237.this version posted May 7, 2020. The copyright holder for this preprint (which was not certified by peer review) is the author/funder, who has granted medRxiv a license to display the preprint in perpetuity. All rights reserved. No reuse allowed without permission.

19. D. F. Gudbjartsson et al., Spread of SARS-CoV-2 in the Icelandic Population. N Engl J Med, NEJMoa2006100-14 (2020).

20. Y. Xu et al., Characteristics of pediatric SARS-CoV-2 infection and potential evidence for persistent fecal viral shedding. Nature Medicine, 1-9 (2020). 


\title{
Supplementary Text for: STeCC: Smart Testing with Contact Counting Enhances Covid-19 Mitigation by Bluetooth App Based Contact Tracing
}

\author{
Hossein Gorji, Markus Arnoldini, David F. Jenny, Wolf-Dietrich Hardt, Patrick Jenny
}

\section{Model}

A model is proposed to compute the numbers of infected people whose infection has not (yet) been detected and the numbers of infected persons with a detected infection ( $n_{i}^{\text {undet }}$ and $\left.n_{i}^{\text {det }}\right)$, respectively (Fig. 1$)$. Note that detected here refers to persons being isolated, which comprises not only those being tested positive, but also those who feel strong symptoms and thus stay in self-quarantine. It is further important to notice that in the case of SARS-CoV2, the undetected infected people are main contributors to the spread of the pandemic [13]. The exact definitions of detected and undetected, as well as those of all other variables and model parameters are found in Table S1. Furthermore, we compute the number of fatalities $\left(n_{d}\right)$ and the number of people who recovered after a detected or an undetected infection. Importantly, we assume that these people will have developed protective immunity and we assume that they cannot be infected again in the considered time frame. The initial susceptible population $n_{s}^{0}$ is naive (i.e. it lacks immunity against the infection) and $n_{s}$ is the number of persons who are susceptible at a given time $t$. In our model we assume that the virus is mainly passed on by undetected asymptomatic and mild symptomatic persons; the detected population with mild symptoms transmits at a much lower rate (because of self-isolation, hygiene precautions in hospitals and/or quarantine). While the model does not consider age dependency, it accounts for higher mortality rates due to temporary shortage of intensive care units. The graph in Fig. 1 (main part) shows the dynamic dependencies. The Covid-19 specific parameters have to be estimated from the available data; their values are listed below. It should be noted, that the implementation of our model allows updating our current estimates with more precise values, as new data come in.

Initially, the entire population is susceptible and can get infected. Infected persons first get exposed and are not infectious before the latency time has passed. Then, they either become asymptomatic or mild symptomatic. Asymptomatic persons eventually recover without symptoms, while the others develop symptoms approximately half a day after the end of the latency time. We assume that persons with mild symptoms isolate themselves approximately one day after onset of symptoms and then either recover or become strong symptomatic, which requires hospitalization. Hospitalized individuals either recover or die. Once $n_{s}$ becomes smaller, which happens quickly without any measures, the infection rate slows down by a factor of $n_{s} / n_{s}^{0}$. This mechanism of slowing down spread of the epidemic due to a shrinking susceptible population is equivalent to herd immunity. It is crucial for the system dynamics that detected persons are isolated (either by self-isolation at home, by hygienic isolation in hospital setting or in other care facilities, or by organized isolation programs for detected infected people, e.g. in hotel rooms) and thus participate at a much smaller rate or not at all in spreading the disease. We assume that these detected infected people have a 10-fold lower likelihood of infecting others than undetected infected people. All this leads to a dynamic system, which is governed 


\begin{tabular}{|c|c|}
\hline terminology & meaning \\
\hline $\begin{array}{l}\text { susceptible } \\
\text { exposed } \\
\text { asymptomatic } \\
\text { pre- and mild sympt. (no self isol.) } \\
\text { mild sympt. (self isol.) } \\
\text { strong symptomatic } \\
\text { deceased } \\
\text { recovered } \\
\text { detected } \\
\text { undetected } \\
\text { transmissive } \\
\text { extreme social distancing } \\
\text { moderate social distancing } \\
\text { mild social distancing }\end{array}$ & $\begin{array}{l}\text { persons of the considered population who are susceptible and thus can potentially get infected } \\
\text { infected persons; can not yet transmit the virus } \\
\text { infected persons without symptoms; can transmit the virus } \\
\text { infected persons with no or mild symptoms; infectious, but not isolated } \\
\text { infected persons with mild symptoms; infectious and isolated } \\
\text { infected persons with strong symptoms and thus hospitalized; isolated } \\
\text { persons who died } \\
\text { persons who recovered } \\
\text { isolated either after positive testing or after falling ill } \\
\text { persons who are either exposed, asymptomatic or mild symptomatic, but were never contained } \\
\text { persons who are either asymptomatic or symptomatic } \\
\mathcal{R}_{0}=0.7 \text {, if no other mitigation measures are applied; the infection rate is reduced by } 71 \% \\
\mathcal{R}_{0}=1.0 \text {, if no other mitigation measures are applied; the infection rate is reduced by } 58 \% \\
\mathcal{R}_{0}=1.6 \text {, if no other mitigation measures are applied; the infection rate is reduced by } 33 \%\end{array}$ \\
\hline \multicolumn{2}{|l|}{ variable } \\
\hline $\begin{array}{l}n_{s} \text { and } n_{s}^{0} \\
n_{e}, \tilde{n}_{e} \text { and } n_{e}^{t o t} \\
n_{i a}, \tilde{n}_{i a} \text { and } n_{i a}^{t o t} \\
n_{i m}, \tilde{n}_{i m} \text { and } n_{i m}^{t o t} \\
n_{m s}, \tilde{n}_{m s} \text { and } n_{m s}^{t o t} \\
n_{s s}, \tilde{n}_{s s} \text { and } n_{s s}^{\text {tot }} \\
n_{d}, \tilde{n}_{d} \text { and } n_{d}^{\text {tot }} \\
n_{r a}, \tilde{n}_{r a} \text { and } n_{r a}^{t o t} \\
n_{r s}, \tilde{n}_{r s} \text { and } n_{r s}^{\text {tot }}\end{array}$ & $\begin{array}{l}\text { numbers of susceptible and initially susceptible persons, respectively } \\
\text { numbers of exposed persons; not tested, tested and in total, respectively } \\
\text { numbers of asymptomatic persons; not tested, tested and in total, respectively } \\
\text { numbers of persons with mild symptoms during first day; not tested, tested and in total, respectively } \\
\text { numbers of persons with mild symptoms after first day; not tested, tested and in total, respectively } \\
\text { numbers of persons with strong symptoms; not tested, tested and in total, respectively } \\
\text { numbers of deceased persons; not tested, tested and in total, respectively } \\
\text { numbers of recovered persons who had no symptoms; not tested, tested and in total, respectively } \\
\text { numbers of recovered persons who had symptoms; not tested, tested and in total, respectively }\end{array}$ \\
\hline $\begin{array}{l}n_{i}^{\text {undet }} \\
n_{i}^{\text {det }}\end{array}$ & $\begin{array}{l}\text { undetected infected persons: } n_{i e}+n_{i a}+n_{i m} \\
\text { detected infected persons: } n_{m s}+n_{s s}+\tilde{n}_{m s}+\tilde{n}_{s s}+\tilde{n}_{i e}+\tilde{n}_{i a}+\tilde{n}_{i m}\end{array}$ \\
\hline $\begin{array}{l}\mathcal{K}, P_{k} \text { and } \mu \\
\mathcal{K}_{0}\end{array}$ & $\begin{array}{l}\text { number of contacts, its probability density and its average, respectively } \\
\text { minimum degree of connectivity in the sub-population }\end{array}$ \\
\hline \multicolumn{2}{|l|}{ parameter } \\
\hline $\begin{array}{l}\alpha \text { and } A(\tau) \\
Q \\
\epsilon \\
\beta_{a} \text { and } \beta_{s} \\
\theta \\
\gamma_{a}, \gamma_{m s} \text { and } \gamma_{s s} \\
\xi_{m s} \text { and } \xi_{s s} \\
k_{e}, k_{a} \text { and } k_{s} \\
\mathcal{R}_{0} \\
\mathcal{R}_{0}^{s y m p} \text { and } \mathcal{R}_{0}^{a s y m} \\
\mathcal{R}_{0}^{w t}, \mathcal{R}_{0}^{n n}, \mathcal{R}_{0}^{S T-A}, \mathcal{R}_{0}^{S T-B} \text { and } \mathcal{R}_{0}^{S T-C} \\
\kappa \\
\zeta \\
\theta^{(s a t)} \text { and } \theta^{(0)} \\
\gamma_{s s}^{(s a t)} \text { and } \gamma_{s s}^{(0)} \\
N \\
N^{-1} \\
\eta \\
C^{(s a t)} / 2.5 \\
m \text { and } k_{C} \\
\gamma \\
T \\
\mathcal{E}, \mathcal{E}^{S T-A}, \mathcal{E}^{S T-B} \text { and } \mathcal{E}^{S T-C} \\
\end{array}$ & $\begin{array}{l}\text { rate coefficient for infection and expected infectiousness } \tau \text { days after infection, respectively } \\
\text { relative infection rate by outside contact (travel) } \\
\text { ratio between infection rate of self-quarantined and non-quarantined symptomatic cases } \\
\text { rate coefficient for latency of asymptomatic and symptomatic cases, respectively } \\
\text { rate coefficient for mortality of hospitalized cases } \\
\text { rate coefficients for recovery } \\
\text { rate coefficients for successively stronger symptoms } \\
\text { rate coefficients accounting for testing } \\
\text { basic reproduction number } \\
\text { basic reproduction number of symptomatic and asymptomatic cases, respectively } \\
\text { reproduction number subject to testing, reshaping the network, STeCC-A, STeCC-B and STeCC-C, respectively } \\
\text { fraction of basic reproduction number related to symptomatic cases } \\
\text { percentage of app users among smart-phone owners } \\
\text { rate coefficient for mortality of hospitalized cases without and with intensive care units, respectively } \\
\text { rate coefficient for recovery of hospitalized cases without and with intensive care units, respectively } \\
\text { testing interval } \\
\text { testing frequency } \\
\text { fraction of false negative test results } \\
\text { fraction of total population for which intensive care units are available } \\
\text { lower and higher cut-off of the connectivity distribution, respectively } \\
\text { difference between the exponent of the power law model and } 2 \\
\text { disease transmissibility } \\
\text { efficacy of contact counting, STeCC-A, STeCC B and STeCC C, respectively }\end{array}$ \\
\hline \multicolumn{2}{|l|}{ operator } \\
\hline $\begin{array}{l}\mathbb{E}(\cdot) \\
\mathcal{P}_{\mathcal{A}} \text { and } \operatorname{Prob}\{\mathcal{A}\}\end{array}$ & $\begin{array}{l}\text { expectation } \\
\text { probability of the event } \mathcal{A}\end{array}$ \\
\hline
\end{tabular}

Table S 1. Terminology and nomenclature of model parameters and variables 
by the following ordinary differential equations:

$$
\begin{aligned}
\dot{n}_{s} & =-\alpha\left(n_{i a} / 2+n_{i m}+\epsilon n_{m s}\right) \frac{n_{s}}{n_{s}^{0}}-Q \frac{n_{s}}{n_{s}^{0}} \\
\dot{n}_{e} & =\alpha\left(n_{i a} / 2+n_{i m}+\epsilon n_{m s}\right) \frac{n_{s}}{n_{s}^{0}}-\left(\beta_{a}+\beta_{s}\right) n_{e}+Q \frac{n_{s}}{n_{s}^{0}}-k_{e} n_{e} \\
\dot{n}_{i a} & =\beta_{a} n_{e}-\gamma_{a} n_{i a}-k_{a} n_{i a} \\
\dot{n}_{i m} & =\beta_{s} n_{e}-\xi_{m s} n_{i m}-k_{s} n_{i m} \\
\dot{n}_{r a} & =\gamma_{a} n_{i a} \\
\dot{n}_{m s} & =\xi_{m s} n_{i m}-\left(\gamma_{m s}+\xi_{s s}\right) n_{m s} \\
\dot{n}_{s s} & =\xi_{s s} n_{m s}-\left(\gamma_{s s}+\theta\right) n_{s s} \\
\dot{n}_{r s} & =\gamma_{s s} n_{s s}+\gamma_{m s} n_{m s} \text { and } \\
\dot{n}_{d} & =\theta n_{s s} .
\end{aligned}
$$

Note that $\epsilon \in[0,1]$ is the transmission reduction factor of the self-isolated individuals. It is assumed that those infected persons who were detected by testing or hospitalized infect much less due to strong isolation and other precautions. Therefore, their effect on the infection rate is neglected here. In order to parametrize the model, besides the rates $\alpha$, $\beta_{a}, \beta_{s}, \gamma_{a}, \gamma_{m s}, \gamma_{s s}, \xi_{m s}, \xi_{s s}$ and $\theta$, also the relative rate $Q$ of infections from outside, i.e., by travel or from the animal world, has to be determined. Further, the initial values of $n_{s}(t), n_{e}(t), n_{i a}(t), n_{i m}(t), n_{r a}(t), n_{m s}(t), n_{s s}(t), n_{r s}(t)$ and $n_{d}(t)$ have to be chosen. The variables $\tilde{n}_{e}(t), \tilde{n}_{i a}(t), \tilde{n}_{i m}(t), \tilde{n}_{r a}(t), \tilde{n}_{m s}(t), \tilde{n}_{s s}(t), \tilde{n}_{r s}(t)$ and $\tilde{n}_{d}(t)$ denote the respective numbers of persons who were tested positive and thus are removed from transmission. The detection rates of exposed $\left(n_{e}\right)$, asymptomatic $\left(n_{i a}\right)$ and mild symptomatic $\left(n_{i m}\right)$ persons due to testing are proportional to $k_{e}, k_{a}$ and $k_{s}$, respectively. These individuals are then accounted for by the respective numbers $\tilde{n}_{e}(t), \tilde{n}_{i a}(t)$ and $\tilde{n}_{i m}(t)$; see Fig. S1. Note that the graph in Fig. S1 is very similar as the one in Fig. 1, except that there is no node for susceptible persons (since by definition a susceptible person can not be detected infected) and that there exist sources due to testing (dotted arrows) instead of sinks. To account for the dynamics with testing, the system (1)-(9) has to be augmented by the ordinary differential equations

$$
\begin{aligned}
\dot{\tilde{n}}_{e} & =-\left(\beta_{a}+\beta_{s}\right) \tilde{n}_{e}+k_{e} n_{e}, \\
\dot{\tilde{n}}_{i a} & =\beta_{a} \tilde{n}_{e}-\gamma_{a} \tilde{n}_{i a}+k_{a} n_{i a}, \\
\dot{\tilde{n}}_{i m} & =\beta_{s} \tilde{n}_{e}-\xi_{m s} \tilde{n}_{i m}+k_{s} n_{i m}, \\
\dot{\tilde{n}}_{r a} & =\gamma_{a} \tilde{n}_{i a}, \\
\dot{\tilde{n}}_{m s} & =\xi_{m s} \tilde{n}_{i m}-\left(\gamma_{m s}+\xi_{s s}\right) \tilde{n}_{m s}, \\
\dot{\tilde{n}}_{s s} & =\xi_{s s} \tilde{n}_{m s}-\left(\gamma_{s s}+\theta\right) \tilde{n}_{s s}, \\
\dot{\tilde{n}}_{r s} & =\gamma_{s s} \tilde{n}_{s s}+\gamma_{m s} \tilde{n}_{m s} \text { and } \\
\dot{\tilde{n}}_{d} & =\theta \tilde{n}_{s s} .
\end{aligned}
$$

The effect of testing is further discussed in Section 3. Next it is described how the parameters can be estimated based on literature data.

\section{Parameter Estimation}

Our devised generalized SEIR model becomes closed once we tune the rate coefficients. These coefficients were computed mainly based on data provided in recently published reports $[6,27]$. Before giving the values for transfer 


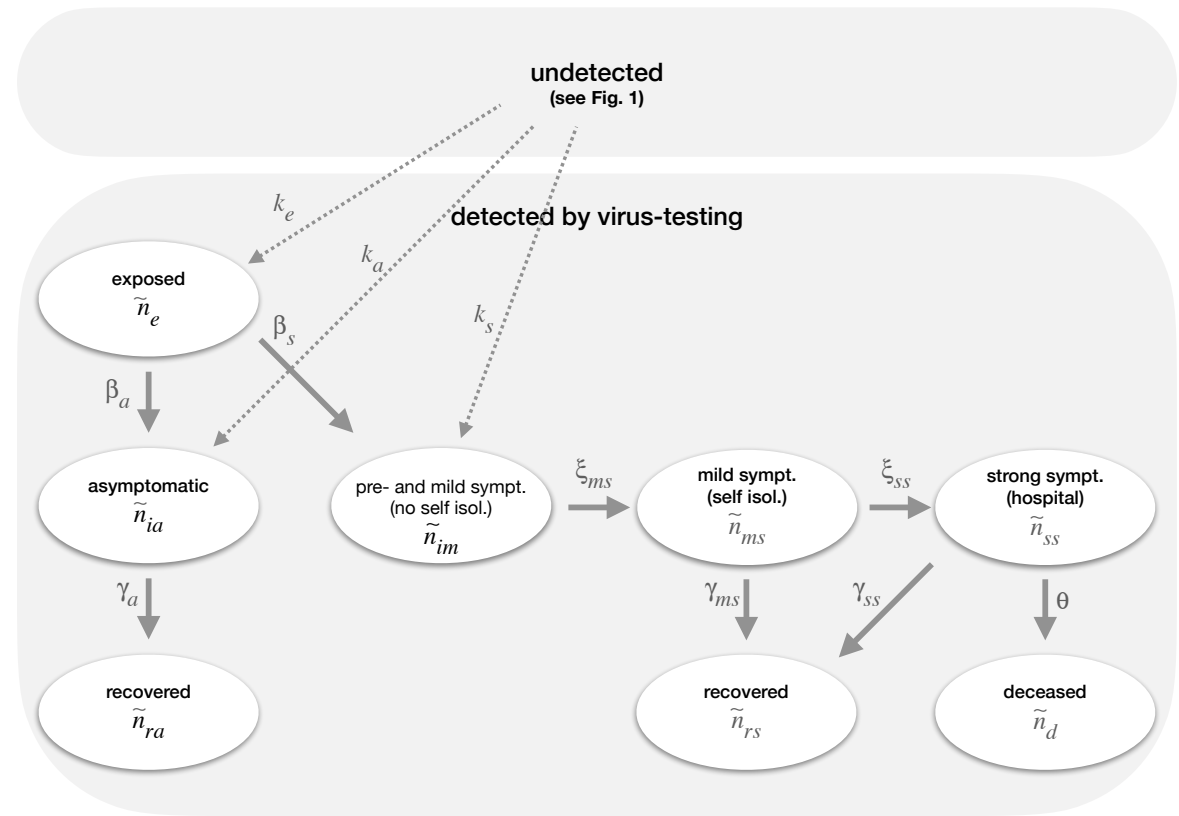

Fig. S 1. Graph showing the dependencies of the compartments describing the dynamics of the positively tested people. 
rates between different compartments, let us analyze the basic reproduction number $\mathcal{R}_{0}$ of this virus infection with $Q=0$ and $n_{s}(t) \approx n_{s}^{0}$. Note that $\mathcal{R}_{0}$ represents "the expected number of secondary cases produced, in a completely susceptible population, by a typical infective individual" [4]. If $\mathcal{R}_{0}$ becomes $<1$, virus spread will decline, and if $\mathcal{R}_{0}>1$, virus spread will increase. To compute $\mathcal{R}_{0}$, we split the dynamics of the infected population into the infection driven propagation $f$ and the remainder $V$, i.e.,

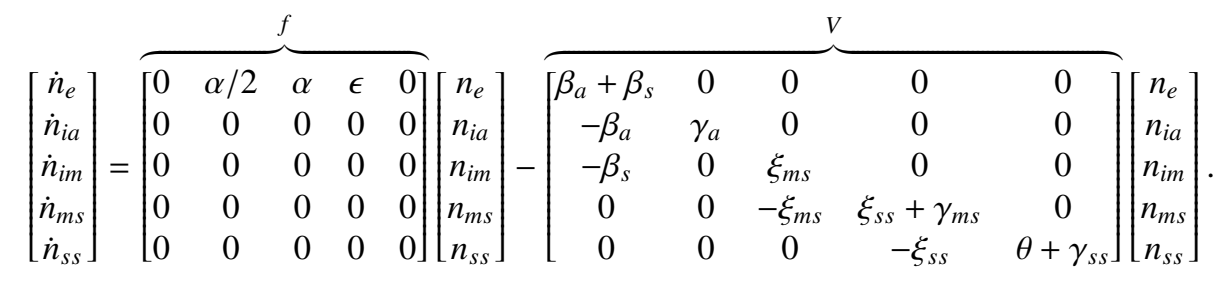

Note that testing is not considered here, i.e., $k_{e}, k_{a}$ and $k_{s}$ are zero. The $\mathcal{R}_{0}$ of this system is the spectral radius of $f V^{-1}$, that is,

$$
\mathcal{R}_{0}=\rho\left(f V^{-1}\right)=\frac{\alpha \beta_{s}}{\beta_{a}+\beta_{s}}\left[\frac{\beta_{a}}{2 \gamma_{a} \beta_{s}}+\frac{1}{\xi_{m s}}+\frac{\epsilon}{\gamma_{m s}+\xi_{s s}}\right] .
$$

By inspecting Eq. (19), we observe that we can move towards a stable state (corresponding to $\mathcal{R}_{0} \leq 1$ ) by reducing the infection rate $\alpha$ via mitigation policies such as social distancing. Importantly, as shown later, $\mathcal{R}_{0}$ can be reduced as well by introducing mass testing, contact tracing, smart testing and subsequent isolation of detected infected individuals.

Next, to clarify our choice of model coefficients, we discuss the rates which appear in transmissive and non-transmissive compartments separately. Finally, the increase of mortality due to lack of intensive care units is modeled.

1. Transmissive: We model the incubation time to be log-normally distributed with mean 5.84 (day) and standard deviation 2.98 (day) $[25,18]$. In accordance with [6] we take the latency time $x_{l}$ such that in average it becomes half a day shorter than the incubation time. Similar to the incubation time, we adopt a log-normal distribution for $x_{l}$ but with mean 5.34 (day) and standard deviation 2.7249 (day). We assume that $1 / 3$ of the cases won't have noticeable symptoms and $2 / 3$ become symptomatic half a day after latency [6]. This leads to $\beta_{a}=\frac{1}{3} \mathbb{E}\left(1 / x_{l}\right)=0.078$ $(1 /$ day), where $\mathbb{E}(\cdot)$ denotes the expectation which gives us the average latency rate. Due to the ratio of $1 / 3$ to $2 / 3$ between asymptomatic and symptomatic cases we get the transfer rate from being exposed to infectious symptomatic as $\beta_{s}=2 \beta_{a}=0.156$ (1/day).

We suppose that it takes around 1 day from onset of symptoms to self-isolation [6]. Since it takes half a day time delay from becoming infectious to symptomatic, we get $\xi_{m s}=1 / 1.5=0.6667$ (1/day).

The average onset to discharge time of clinical cases is around 22 days [27]. We assume that for mild-symptomatic cases the onset to recovery time would be half of this amount, i.e., around 11 days. Therefore the average recovery time from end of the latency period becomes 11.5 days for mild-symptomatic cases. We set the same recovery time for asymptomatic cases which leads to $\gamma_{a}=1 / 11.5=0.087(1 /$ day $)$.

A range of values have been suggested for infectiousness of asymptomatic cases; one finds 0.1 in [7], 2/3 in [6] and 1 in [12]. We assume that the asymptomatic cases are 50\% less infectious. Furthermore we consider the self quarantined patients to be $90 \%$ less infectious, i.e., $\epsilon=0.1$ is adopted. To compute the infection rate $\alpha$, we assume $\mathcal{R}_{0}=2.4$ [6]. Following Eq. (19), the infection rate becomes $\alpha=0.6711$ (1/day). Since the basic 
reproduction number is the most important single parameter of the system, we performed sensitivity studies by changing $\mathcal{R}_{0}$ [14].

2. Non-transmissive: The mean delay time from appearance of symptoms to hospitalization has been reported to be around 11 days [27]. However, $80 \%$ of symptomatic cases would not require hospitalization [15]. For those who develop strong symptoms, the delay from self-isolation to hospitalization then becomes $11-1=10$ days. Hence we get $\xi_{s s}=0.2 \times 1 / 10=0.02(1 /$ day $)$ and $\gamma_{m s}=0.8 \times 1 / 10=0.08(1 /$ day $)$. Note that the latter gives onset to recovery time of 11 days for mild cases consistent with our earlier assumption.

The average hospital treatment time is 11 days $[27,6]$. In case of availability of intensive care units we assume that $20 \%$ of hospitalized cases die [15]. Accordingly, we get $\gamma_{s s}^{(0)}=0.8 \times 1 / 11=0.0727(1 /$ day $)$ and $\theta^{(0)}=0.2 \times 1 / 11=0.0182(1 /$ day $)$.

3. Fatality increase: We assume that the case fatality ratio increases by two-fold in saturation of the health system. This is justified by noting that the case fatality ratio has increased from approximately $5 \%$ in China [2] to roughly $10 \%$ in Wuhan while it was the epicentre of the outbreak [20]. By taking this factor into account, and assuming that the average time of hospital treatment remains 11 days, we can compute the death rate of hospitalized cases once saturation of intensive care units is reached as $\theta^{(\text {sat })}=0.4 \times 1 / 11=0.0364$ (1/day). Note that consistently one obtains $\gamma_{s s}^{(s a t)}=0.6 \times 1 / 11=0.0546(1 /$ day $)$. It is assumed that there exist eight intensive care beds per 100 '000 persons [6] and that $40 \%$ of the hospitalized cases need such treatment. Accordingly, saturation is reached once the number of hospitalized cases, i.e., $n_{s s}$, exceeds $C^{(\text {sat })}=0.02 \%$ of the total population.

The adjusted rate

$$
\theta\left(n_{s s}^{\text {tot }}\right)=\frac{n_{s}^{0}}{n_{s s}^{\text {tot }}}\left(\min \left\{C^{(\mathrm{sat})}, \frac{n_{s s}^{\text {tot }}}{n_{s}^{0}}\right\} \theta^{(0)}+\max \left\{0, \frac{n_{s s}^{\text {tot }}}{n_{s}^{0}}-C^{(\mathrm{sat})}\right\} \theta^{\text {(sat) })}\right.
$$

then quantifies the death rate of hospitalized cases as the weighted average of $\theta^{(0)}$ and $\theta^{(\text {sat })}$; the consistently adjusted recovery rate becomes

$$
\gamma_{s s}\left(n_{s s}^{\text {tot }}\right)=\gamma_{s s}^{(0)}+\theta^{(0)}-\theta\left(n_{s s}^{\text {tot }}\right)
$$

All estimates here are summarized in Table S2; note that these values can easily be adapted, if more reliable data becomes available. The resulting parameter values for our base case are provided in Table S3.

Figure $2 \mathrm{~A}$ shows the model results without mitigation for a period of 600 days with $\mathcal{R}_{0}=2.4$. Results with $\mathcal{R}_{0} \in$ $\{1.9,2.9\}$ are shown in Fig. S2A,B. Dashed lines represent the immune plus deceased plus infected $\left(n_{s}^{0}-n_{s}\right)$, dashdotted lines the infected $\left(n_{i}^{\text {undet }}+n_{i}^{\text {det }}\right)$ and solid lines the deceased $\left(n_{d}^{\text {tot }}\right)$ population. For bigger values of $\mathcal{R}_{0}$, a larger immune population is needed to achieve herd immunity (right half of the graphs), and the peak in the number of infections is higher and sharper.

The plot in Fig. S2C shows the case with $\mathcal{R}_{0}=2.4$ without intensive care unit limitation; compare with Fig. 2A, which shows the same case with intensive care unit limitation (our base case). In both cases $87 \%$ of the population will become immune, which compares well with $81 \%$ infected people predicted by [6] for the UK and US populations in the absence of mitigation plans. Without intensive care the chance of dying is roughly twice as high for strong symptomatic people than with proper treatment $(4.6 \%$ vs. $2.3 \%)$. While these numbers are subject to errors (mainly due to 


\begin{tabular}{|c|c|c|c|}
\hline probability & conditional on & expression & base case value \\
\hline$\rightarrow$ pre- and mild sympt. (no self isol.) & asympt. & $S^{(m)}$ & $2 / 3,[1 / 2,2 / 3]$ \\
\hline$\rightarrow$ strong sympt. (hospit.) & mild sympt. (self isol.) & $S^{(s)}$ & $1 / 5$ \\
\hline$\rightarrow$ deceased & hospit.; with icu & $M^{(0)}$ & $1 / 5$ \\
\hline$\rightarrow$ deceased & hospit.; no icu & $M^{(\text {sat })}$ & $2 / 5$ \\
\hline \multicolumn{4}{|l|}{ char. time scale (days) } \\
\hline$\rightarrow$ pre- and mild sympt. (no self isol.) & exposed & $S^{(m)} \beta_{s}^{-1}$ & 4.27 \\
\hline$\rightarrow$ mild sympt. (self isol.) & pre- and mild sympt. (no self isol.) & $t_{i m}=\xi_{m s}^{-1}$ & 1.5 \\
\hline$\rightarrow$ strong sympt. (hospit.) & mild sympt. (self isol.) & $t_{m s}=S^{(s)} \xi_{s s}^{-1}$ & 10 \\
\hline$\rightarrow$ deceased & str. sympt. & $M^{(0, s a t)} / \theta^{(0, s a t)}$ & 11 \\
\hline$\rightarrow$ recovered & asymptomatic & $t_{i a}=\gamma_{a}^{-1}$ & 11.5 \\
\hline \multicolumn{4}{|l|}{ further parameters } \\
\hline basic reproduction number & & $\overline{\mathcal{R}_{0}}$ & 2.4 \\
\hline infection rate reduction factor & mild sympt. (self isol.) & $\epsilon$ & 0.1 \\
\hline rel. intensive care capacity & & $C^{(s a t)} / 2.5$ & 0.00008 \\
\hline \multicolumn{4}{|l|}{ social distancing, contact tracing and testing } \\
\hline infection rate reduction factor & social distancing & $\lambda$ & 0 \\
\hline testing frequency (1/days) & testing & $N^{-1}$ & 0 \\
\hline testing process time (days) & testing & $\tau_{\text {proc }}$ & 1 \\
\hline fraction of false negative test results & testing & $\eta$ & $0.05,\{0.5,0.15,0.25\}$ \\
\hline success rate of contact tracing & contact tracing & $\zeta$ & {$[0.3,1]$} \\
\hline fraction of exposed people who develop no symptoms & contact tracing & $r_{1}$ & {$[1 / 3,1 / 2]$} \\
\hline fraction of exposed people who develop no symptoms & contact tracing & $r_{2}$ & {$[0.1,0.5]$} \\
\hline
\end{tabular}

Table S 2. Estimations made for the model closure, social distancing, contact tracing and testing.

\begin{tabular}{|c|c|}
\hline parameter & value \\
\hline$\alpha$ & 0.670 (1/day) \\
\hline$\epsilon$ & 0.1 \\
\hline$\beta_{a}$ & 0.078 (1/day) \\
\hline$\beta_{s}$ & 0.156 (1/day) \\
\hline$\gamma_{a}$ & 0.087 (1/day) \\
\hline$\xi_{m s}$ & 0.667 (1/day) \\
\hline$\gamma_{m s}$ & 0.08 (1/day) \\
\hline$\xi_{s s}$ & 0.02 (1/day) \\
\hline$\gamma_{s s}^{(0)}$ & 0.072 (1/day) \\
\hline$\theta^{(0)}$ & 0.0182 (1/day) \\
\hline$\theta^{(\text {sat })}$ & 0.0364 (1/day) \\
\hline initial condition & value \\
\hline $\begin{array}{c}n_{s}^{0} \\
n_{e}(0)\end{array}$ & $\begin{array}{l}\text { 6'384'631'490 (world population outside of China) } \\
\text { 1'000 }\end{array}$ \\
\hline
\end{tabular}

Table S 3. List of estimated parameters and initial values. Note that our model allows to easily replace any of these parameters by more precise estimates, as more data become available. The initial values of all numbers except $n_{e}$ are set to zero.

uncertainties in the parameter values and efforts to increase intensive care and respirator availability), it can be expected that the relevant dynamics is captured to a high degree. If the results are regarded with respect to the base case, much insight can be gained, e.g. how social distancing, mass testing and smart testing can be combined most effectively. 


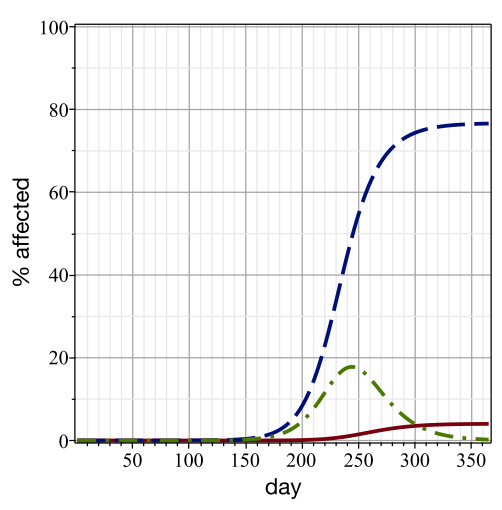

$\mathcal{R}_{0}=1.9$; with ICU capacity limit

(A)

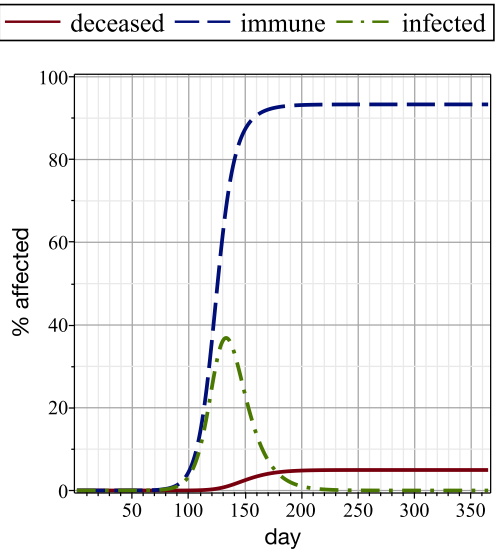

$\mathcal{R}_{0}=2.9$; with ICU capacity limit

(B)

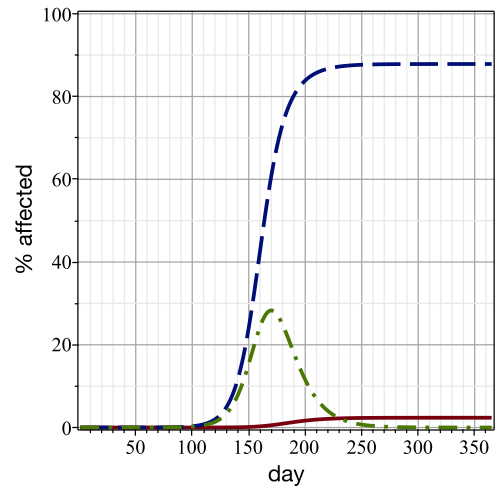

$\mathcal{R}_{0}=2.4 ;$ without ICU capacity limit (C)

Fig. S 2. Alternative model outcomes when changing $\mathcal{R}_{0}$ of the pandemic, or relaxing the assumption that ICU beds are limiting. Dashed blue lines represent recovered and deceased, dashed-dotted green lines infected, and solid red lines deceased people. Changing $\mathcal{R}_{0}$ to (A) 1.9 and (B) 2.9 changes the outcomes quantitatively, but does not change the overall picture. (C) Model outcome if the assumption that full ICUs increase the death rate is dropped.

\section{Mass Testing - How the Number of Tests Relates to $\mathcal{R}_{\mathbf{0}}$}

Here we analyze how many tests are needed to mitigate the Covid-19 pandemic if no other mitigation strategies were applied. If we can use more tests than that, $\mathcal{R}_{0}$ will drop below one, the incidence would decline and the pandemic would eventually end, even if no vaccines or infection therapies become available. Therefore we studied how $\mathcal{R}_{0}$ varies once confirmed cases get isolated (in addition to self-quarantined and hospitalized individuals). Of particular value is the relationship between $\mathcal{R}_{0}$ and the interval of testing the susceptible population (i.e., the frequency of testing needed for reducing $\mathcal{R}_{0}$ below one). Thereby we can determine the key technical parameter of interest, i.e., the number of tests per 100'000 people that must be tested per day in order to achieve the desired $\mathcal{R}_{0}$ value; we chose $\mathcal{R}_{0}=1$ as the target value for our analyses (if not indicated otherwise), which would suffice to keep the number of infected people constant.

To be realistic, we suppose that the processing time $\tau_{\text {proc }}$ of mass testing would be somewhere between half a day and two days. Furthermore, a fraction $\eta=0.05$ of false negative test results is taken into account [17]. It should be noted, however, that this is a rough estimate. The use of standards allows for very high reproducibly of the virus RNA detection results even between different laboratories [3]. The true rate of false negatives and false positives is currently not known. We assume that a false negative rate of $5 \%$ is a conservative estimate. The current virus RNA testing capacities in continental Europe reach up to 230 tests per 100'000 people per day (e.g. in Switzerland). If equipment and supplies are not limiting for testing, e.g. by using a quantitative polymerase chain reaction (qPCR) method ${ }^{1}$, we estimate that up to around 1'000 samples within a time frame of eight hours can be analyzed per machine. Mass testing (i.e., if $>500-1^{\prime} 000$ tests per 100'000 perople per day would be required) could be realizable by taking advantage of next-generation RNA extraction, reverse transcription and sequencing (combined with reverse transcription and PCR) to detect the virus RNA of infected people. For example, in [11] a massively parallel diagnostic assay is described

\footnotetext{
${ }^{1}$ https ://www.roche.com/media/releases/med-cor-2020-03-13.htm
} 
for testing up to 19’200 patient samples per work flow. In principle, such very high throughput approaches can be parallelized (and potentially optimized) to provide millions of tests per day. In reality, the logistics of collecting these millions of samples would however be a major hurdle. First, we want to assess how many tests were indeed necessary to stop the virus spread (i.e., to reach $\mathcal{R}_{0} \leq 1$ if no other mitigation strategies were applied).

Obviously, the scenario that the whole susceptible population is tested perfectly at once would lead to a trivial disease free state. However, this is an unrealistic scenario, not only because of a lack of test capacity, but also due to logistic and compliance concerns. Therefore, it is only realistic to assume that individuals would be tested at different schedules. Let us consider a situation where each person is tested once every $N$ days. Note that this is equivalent to testing a random fraction of $1 / N$ of the susceptible population every day. Therefore we focus on the set $\{1, \ldots, N\}$ of days. An individual is infected at some random time $x$. To characterize $x$, we assume that the likelihood of getting infected does not vary much during these days, which is justified if testing is applied at an intensity such that $\mathcal{R}_{0} \approx 1$. Hence $x$ becomes uniformly distributed in the interval $[1, N]$. The time delay between infection and detection would then be $\tau_{d e t}=N-x+\tau_{\text {proc }}$. Finally, we sample the latency time $x_{l}$ from a log-normal distribution with $5.34 \pm 2.7249$ (see Section 2).

Intuitively, by conducting mass testing on individuals who are neither self-quarantined nor hospitalized, positive cases will be detected from exposed, asymptomatic and mild-symptomatic compartments. To quantify each detection rate, it is essential to compare detection time versus the latency period; therefore we consider the effect of testing on these three compartments individually:

1. Exposed: Once testing occurs during the latency period of an infected individual, they would be detected from the exposed compartment. This translates into an event set $\mathcal{A}: \tau_{\text {det }} \leq x_{l}$. The rate of detecting individuals by testing from the exposed population then reads

$$
k_{e}=(1-\eta) \mathcal{P}_{\mathcal{A}} \mathbb{E}_{\mathcal{A}}\left[\tau_{\text {det }}^{-1}\right] \text {, }
$$

where $P_{\mathcal{A}}$ and $\mathbb{E}_{\mathcal{A}}$ denote frequency of such events and conditional expectation, respectively.

2. Mild-symptomatic: Once testing occurs after the latency period, one has to distinguish between two types of infection developments. According to our setting, two thirds of the infected individuals would develop symptoms that will lead them to self-isolate. Please note, that the fraction of infected individuals that remain asymptomatic may range between 33\%-50\% [6, 7, 24]; see Table S2. Therefore, we have also tested scenarios where $60 \%$ or $50 \%$ of infected will will progress to develop symptoms; see Fig. S5. These individuals may in fact turn to testing centers in order to detect the virus and incentivize the decision for self-quarantine. They can be detected by testing and therefore sent into quarantine in the span of one and a half days after becoming infectious. The relevant event set is $\mathcal{B}:\left(\tau_{\text {det }} \geq x_{l}\right) \cap\left(\tau_{\text {det }} \leq\left(x_{l}+3 / 2\right)\right)$, from which one obtains

$$
k_{s}=\frac{2}{3}(1-\eta) \mathcal{P}_{\mathcal{B}} \mathbb{E}_{\mathcal{B}}\left[\tau_{\text {det }}^{-1}\right]
$$

for the test detection rate of mild-symptomatic persons.

3. Asymptomatic: This is arguably the most important group to consider, because they will not know that they are infected and they can make up as much as $50 \%$ of the entire group of the infected people. Importantly, they are very hard to identify using the current mitigation strategies, including app based contact tracing [7]. We will discuss this in more detail, below. Testing may catch asymptomatic cases. These individuals won't have 
symptoms and would then recover after 11.5 days. In their first one and a half days they share same the time-line as mild symptomatic ones. Therefore, in this scenario two event sets $\mathcal{B}:\left(\tau_{d e t} \geq x_{l}\right) \cap\left(\tau_{d e t} \leq\left(x_{l}+3 / 2\right)\right)$ and $C:\left(\left(\tau_{\text {det }} \geq\left(x_{l}+3 / 2\right)\right) \cap\left(\tau_{\text {det }} \leq\left(x_{l}+11.5\right)\right)\right.$ become relevant. We obtain the detection rate from the asymptomatic compartment as

$$
k_{a}=\frac{1}{3}(1-\eta) \mathcal{P}_{\mathcal{B}} \mathbb{E}_{\mathcal{B}}\left[\tau_{\text {det }}^{-1}\right]+(1-\eta) \mathcal{P}_{C} \mathbb{E}_{C}\left[\tau_{\text {det }}^{-1}\right]
$$

Before finding the map from testing frequency $N^{-1}$ to $\mathcal{R}_{0}$, we need to find out how $\mathcal{R}_{0}$ varies with respect to the test detection rates $k_{e}, k_{a}$ and $k_{s}$. Hence, let us define $\mathcal{R}_{0}^{w t}$ as the reproduction number subject to testing. To compute $\mathcal{R}_{0}^{w t}$, the main dynamics of the infected population, which can be described by $\left[n_{e}(t), n_{i a}(t), n_{i m}(t), n_{m s}(t), n_{s s}(t), \tilde{n}_{e}(t), \tilde{n}_{i a}(t), \tilde{n}_{i m}(t), \tilde{n}_{m s}(t), \tilde{n}_{s s}(t)\right]^{T}$, is split into the rate of appearance $f$ of new infected individuals and transfer $\tilde{V}$ of infected ones across different compartments, i.e.,

$$
f=\left[\begin{array}{cccccccccc}
0 & \alpha / 2 & \alpha & \epsilon & 0 & 0 & 0 & 0 & 0 & 0 \\
0 & 0 & 0 & 0 & 0 & 0 & 0 & 0 & 0 & 0 \\
0 & 0 & 0 & 0 & 0 & 0 & 0 & 0 & 0 & 0 \\
0 & 0 & 0 & 0 & 0 & 0 & 0 & 0 & 0 & 0 \\
0 & 0 & 0 & 0 & 0 & 0 & 0 & 0 & 0 & 0 \\
0 & 0 & 0 & 0 & 0 & 0 & 0 & 0 & 0 & 0 \\
0 & 0 & 0 & 0 & 0 & 0 & 0 & 0 & 0 & 0 \\
0 & 0 & 0 & 0 & 0 & 0 & 0 & 0 & 0 & 0 \\
0 & 0 & 0 & 0 & 0 & 0 & 0 & 0 & 0 & 0 \\
0 & 0 & 0 & 0 & 0 & 0 & 0 & 0 & 0 & 0
\end{array}\right]
$$

and

$$
\tilde{V}=\left[\begin{array}{cccccccccc}
\beta_{a}+\beta_{s}+k_{e} & 0 & 0 & 0 & 0 & 0 & 0 & 0 & 0 & 0 \\
-\beta_{a} & \gamma_{a}+k_{a} & 0 & 0 & 0 & 0 & 0 & 0 & 0 & 0 \\
-\beta_{s} & 0 & \xi_{m s}+k_{s} & 0 & 0 & 0 & 0 & 0 & 0 & 0 \\
0 & 0 & -\xi_{m s} & \xi_{s s}+\gamma_{m s} & 0 & 0 & 0 & 0 & 0 & 0 \\
0 & 0 & 0 & -\xi_{s s} & \theta+\gamma_{s s} & 0 & 0 & 0 & 0 & 0 \\
-k_{e} & 0 & 0 & 0 & 0 & \beta_{a}+\beta_{s} & 0 & 0 & 0 & 0 \\
0 & -k_{a} & 0 & 0 & 0 & -\beta_{a} & \gamma_{a} & 0 & 0 & 0 \\
0 & 0 & -k_{s} & 0 & 0 & -\beta_{s} & 0 & \xi_{m s} & 0 & 0 \\
0 & 0 & 0 & 0 & 0 & 0 & 0 & -\xi_{m s} & \xi_{s s}+\gamma_{s s} & 0 \\
0 & 0 & 0 & 0 & 0 & 0 & 0 & 0 & -\xi_{s s} & \theta+\gamma_{s s}
\end{array}\right],
$$

which leads to

$$
\mathcal{R}_{0}^{w t}=\rho\left(f \tilde{V}^{-1}\right)=\frac{\alpha \beta_{s}}{\beta_{a}+\beta_{s}+k_{e}}\left[\frac{\beta_{a}}{2\left(\gamma_{a}+k_{a}\right) \beta_{s}}+\frac{1}{\xi_{m s}+k_{s}}\left(1+\frac{\epsilon \xi_{m s}}{\gamma_{m s}+\xi_{s s}}\right)\right] .
$$

It is important to emphasize that in contrast to social distancing the effect of testing on the reproduction number is not through reducing the infection rate (i.e., $f$ remains the same), but rather through transfer of infected individuals to quarantine, either at home or in hotel rooms, or in care units with strict hygiene barriers, all of which would reduce the likelihood of transmission (i.e., changing $V$ to $\tilde{V}$ ). 
Using Monte-Carlo to estimate the test detection rates given by Eqs. (22) and (23) one can compute the ratio $\mathcal{R}_{0}^{w t} / \mathcal{R}_{0}$ with respect to the testing frequency $N^{-1}$. The plots in Figs. S3A,B show the number of tests required to reach $\mathcal{R}_{0}=1$. It depends on the time from sampling to result (note: we assume immediate notification and immediate implementation of quarantine measures upon notification) and on the false negative rate (5\% in Fig. S3A and 15\% in Fig. S3B). The horizontal green dashed lines indicate $\mathcal{R}_{0}^{w t}=1$, if the virus reproduction rate in the case without any mitigation is $\mathcal{R}_{0}=2.4$.

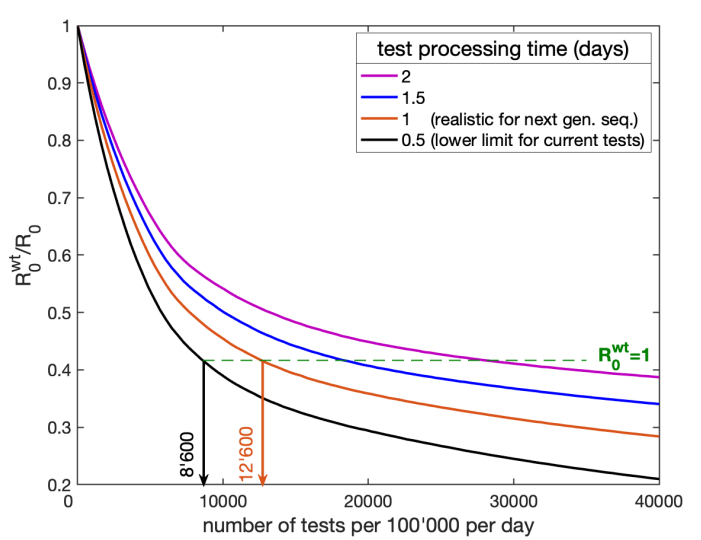

(A)

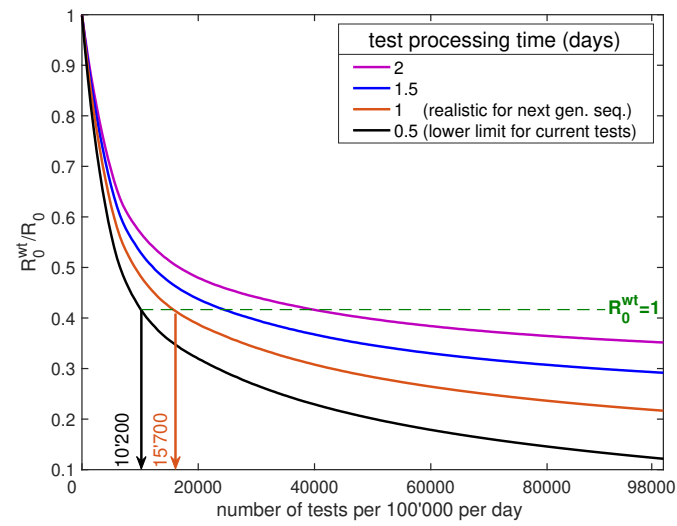

(B)

Fig. S 3. Mass testing: The number of tests required to reach $\mathcal{R}_{0}=1$ depends on the time from sampling to result. A mitigation strategy relying on mass testing alone is assumed and we computed the number of tests performed per day, which are needed to achieve a particular test-speed dependent $\mathcal{R}_{0}^{w t} / \mathcal{R}_{0}$ ratio; for (A) $5 \%$ and (B) $15 \%$ false negative test results are assumed. The green lines indicate $\mathcal{R}_{0}^{w t}=1$. Test speeds were: 0.5 days (black line), 1 day (orange line), 1.5 days (blue line) and 2 days (purple line).

One can see for example that reducing the initial $\mathcal{R}_{0}=2.4$ to one would require to test the entire sceptible population roughly once every eight days, if the false negative rate is $5 \%$, if testing results are available after one day and quarantine of the detected individuals commences immediately. Corresponding results are depicted in Fig. S4B, which shows model outcomes if $\mathcal{R}_{0}$ is reduced to one by testing $12^{\prime} 600$ per $100^{\prime} 000$ people per day. Note that this corresponds to a testing interval of 7.92 days or equivalently to a fraction of $1 / 7.92$ which has to be tested every day. Figure S4A shows the outcome when social distancing is applied to reduce $\mathcal{R}_{0}$ to one, which is equivalent to a $58 \%$ reduction of the infection rate. We define this as moderate social distancing; see Table S1. By comparing Figs. S4A and $\mathrm{S} 4 \mathrm{~B}$ one observes that mass testing and social distancing yield qualitatively equivalent results. For half a day delay time the testing interval can be increased to roughly twelve days and for a delay time of one and a half days it would be five days. This information is important for optimizing technical development decisions. The overall testing capacity needs to be larger, if the testing method requires more time (Fig. 3) or if delays in case-notification or implementation of quarantine measures occur. This could be justified, if a low-cost technique (such as next generation sequencing) could be devised [11]. Alternatively, one could aim for fewer tests, if they are completed in less than half a day. If combined with social distancing, the basic reproduction number can be reduced by the factor $(1-\lambda) \mathcal{R}_{0}^{w t} / \mathcal{R}_{0}$, where $\lambda \in[0,1]$ is the intensity of social distancing $(\lambda=0$ means no social distancing and $\lambda=1$ means complete isolation of everybody). 

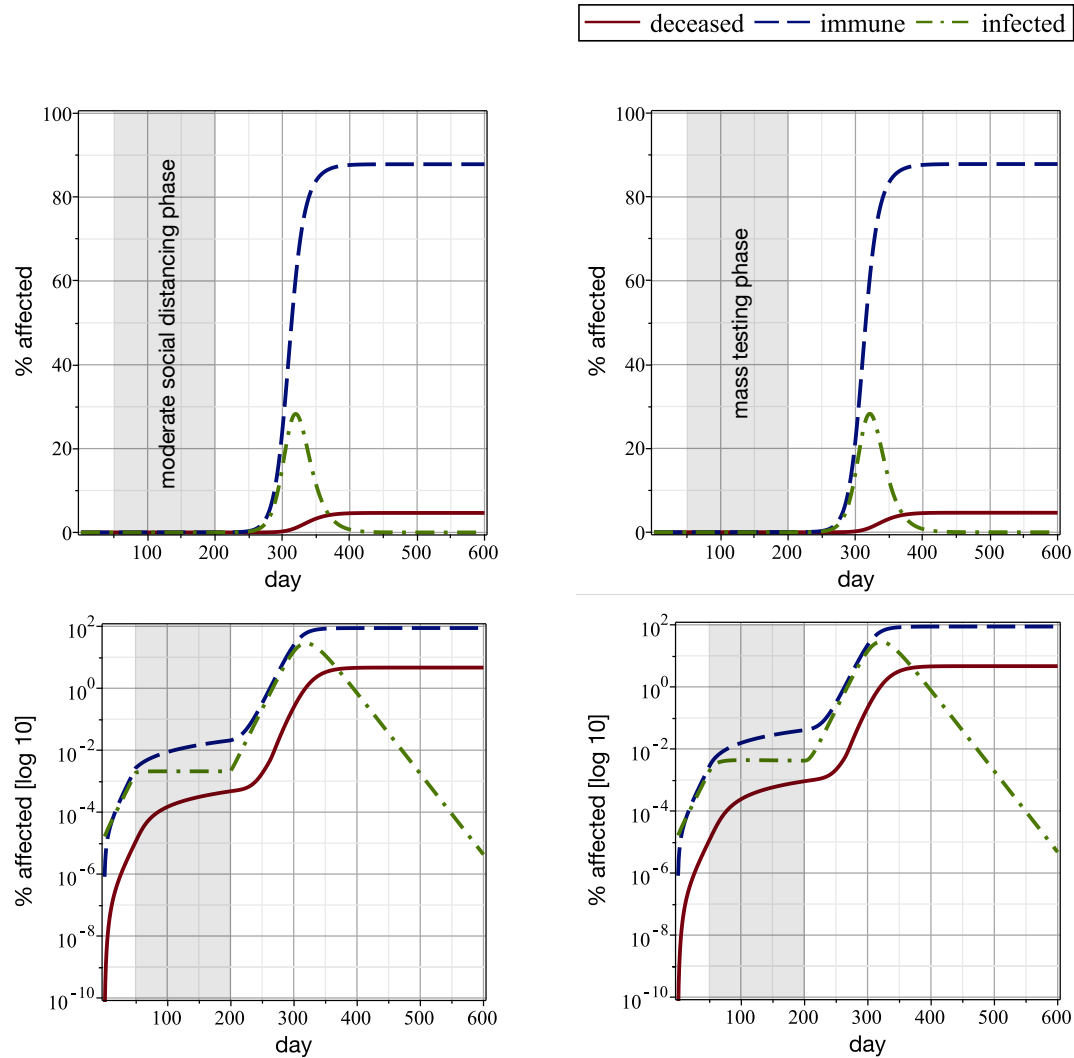

moderate social distancing

$$
\left(\mathcal{R}_{0}=1\right)
$$

(A)

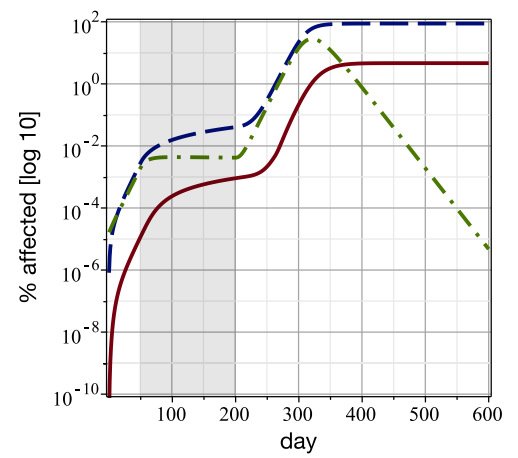

mass testing 12 '600 per 100 '000 per day

$\left(\mathcal{R}_{0}=1\right)$

(B)

Fig. S 4. Moderate social distancing vs. mass testing: Model outcomes if $\mathcal{R}_{0}$ is reduced to 1 by mass testing or social distancing. (A) Moderate social distancing $\left(\mathcal{R}_{0}=1\right.$, equivalent to a $58 \%$ reduction in infection rate) and (B) testing $12^{\prime} 600$ per 100 ' 000 people per day yield qualitatively equivalent results. 


\section{Contact Tracing - How Size and Infectiousness of the Asymptomatic Population Relates to $\mathcal{R}_{0}$}

Contact tracing has been proposed to slow down or even stabilize the pandemic $[7,10,8]$. The strategy is that symptomatic individuals who go into self quarantine will use an App to alert all proximity contacts of the past two weeks. Subsequently, these identified individuals self-isolate themselves. For the following analysis we assume that the notice-to-quarantine time delay is negligible $[7,10]$.

To analyze the effect of contact tracing on the basic reproduction number, the expected infectiousness $A(\tau)$ at time $\tau$ after infection plays a central role. By knowing $A(\tau)$ we can extract the basic reproduction number as

$$
\mathcal{R}_{0}=\int_{0}^{\infty} A(\tau) d \tau
$$

Note that the above equation is consistent with our previous computation of $\mathcal{R}_{0}$ in the linear regime [4]. In our compartmental setting shown in Fig. 1 one obtains the expression

$$
A(\tau)=\sum_{i \in \mathcal{S}_{c}} \mathcal{P}_{i} A_{i}(\tau)
$$

where $\mathcal{P}_{i}$ is the probability that an infected individual is in compartment $c_{i}, \mathcal{S}_{c}=\{s, e, i a, r a, i m, m s, r s, s s, d\}$ is the index set of all compartments and $A_{i}(\tau)$ denotes the infectiousness at time $\tau$ after infection, if the individual is in $c_{i}$. Correspondingly, we denote the time spent in each compartment as $t_{i \in \mathcal{S}_{c}}$. From Eqs. (28) and (29), if we assume that infectiousness is constant inside each compartment, we obtain

$$
\mathcal{R}_{0}=\overbrace{\alpha r_{1} r_{2} t_{i a}}^{\mathcal{R}_{0}^{a s y m}}+\overbrace{\alpha\left(1-r_{1}\right)\left(t_{i m}+\frac{1}{10} t_{m s}\right)}^{\mathcal{R}_{0}^{\text {sym }}},
$$

where $r_{1}=1-S^{(m)}$ is the fraction of exposed people who develop no symptoms (thus remain asymptomatic), and $r_{2}$ is the factor by which asymptomatic people are less infectious than symptomatic ones. For both $r_{1}$ and $r_{2}$ different values are suggested in the literature. For $r_{1}$ one finds $1 / 3$ in [6], 0.4 in [7] and 0.5 in [24], and for $r_{2}$ one finds 0.1 in [7], 2/3 in [6] and 1 in [12]. Based on these published numbers we consider $r_{1} \in[0.3,0.5]$ and $r_{2} \in[0.1,0.5]$. From Eq. (30) with our base case values $r_{1}=1 / 3$ and $r_{2}=0.5$ one obtains $\alpha=0.67$ (1/day), which is consistent with our previous parameter estimation. Based on our previous assumptions and on the values in Table S2 we obtain $t_{i a}=\gamma_{a}^{-1}=11.5$ days, $t_{i m}=\xi_{m s}^{-1}=1.5$ days and $t_{m s}=S^{(s)} \xi_{s s}^{-1}=10$. Interesting here is the fraction

$$
\kappa=\frac{\mathcal{R}_{0}^{\text {sym }}}{\mathcal{R}_{0}}=\frac{\left(1-r_{1}\right)\left(t_{i m}+t_{m s} / 10\right)}{r_{1} r_{2} t_{i a}+\left(1-r_{1}\right)\left(t_{i m}+t_{m s} / 10\right)}
$$

of infected people who got infected by symptomatic cases, since this is the maximum relative reduction of $\mathcal{R}_{0}$ which can be achieved by tracing contacts of symptomatic individuals (by classical contact tracing or by using an app) with a success rate of $\zeta=1$. Ignoring secondary infections (their probability becomes around $0.8 \%$ ) we obtain the approximation

$$
\mathcal{R}_{0}^{c t} \approx[1-\zeta \kappa] \mathcal{R}_{0}
$$

for the basic reproduction number, if contact tracing is employed. Figures S5A and S5B show the performance of contact tracing for different $r_{1^{-}}, r_{2^{-}}$and $\zeta$-values. The numbers attached to the isolines refer to the ratio $\mathcal{R}_{0}^{w t} / \mathcal{R}_{0}$, and 


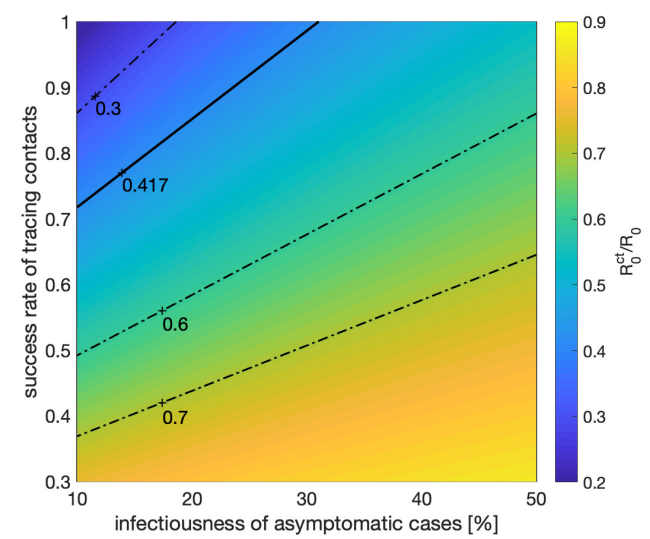

(A)

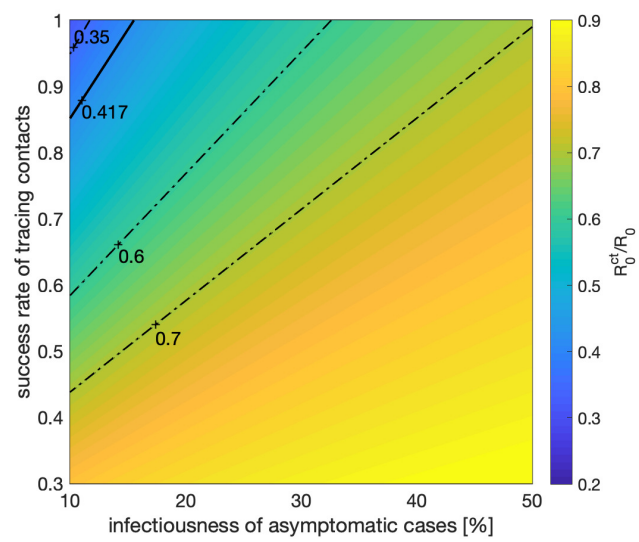

(B)

Fig. S 5. Contact tracing: Effectiveness of contact tracing as function of relative infectiousness of asymptomatic cases $\left(r_{2}\right)$ and the success rate of contact tracing $(\zeta)$. (A): $33 \%$ of infected ones are asymptomatic $\left(r_{1}=0.33\right)$; (B): $50 \%$ of infected ones are asymptomatic $\left(r_{1}=0.5\right)$. The numbers attached to the isolines refer to the ratio $\mathcal{R}_{0}^{w t} / \mathcal{R}_{0}$, and the bold contours depict combinations of $r_{2}$ and $\zeta$ for which $\mathcal{R}_{0}$ is reduced from 2.4 to $\mathcal{R}_{0}^{c t}=1$. In all computations, notice-to-quarantine time was neglected and it was assumed that quarantined contacts are not infectious.

the bold contours depict combinations of $r_{2}$ and $\zeta$ for which $\mathcal{R}_{0}$ is reduced from 2.4 to $\mathcal{R}_{0}^{c t}=1$. One can conclude that the effect of contact tracing, if only applied to identify contacts with symptomatic persons, is limited to optimistic assumptions concerning the parameters dictating the Covid-19 pandemic and strongly depends on size and infectiousness of the asymptomatic population relative to size and infectiousness of the symptomatic one. Even if the most optimistic assumptions would hold, more substantial reductions of $\mathcal{R}_{0}$ would be desirable in order to accelerate the end of the pandemic (e.g. even in the absence of effective therapies or vaccines). For our base parameters of $r_{1}=1 / 3$ and $r_{2}=1 / 2$, the contact tracing alone would not lead to basic reproduction number of one (see Fig. S 5A at 50\% infectiousness).

\section{Smart Testing - How Selectivity Relates to $\mathcal{R}_{0}$}

Here it is studied how the number of required tests can be reduced, if one is able to identify (and propose testing to) a subpopulation with a prevalence higher than the overall population.

Without any additional knowledge, to achieve the discussed detection rates $k_{e}, k_{a}$ and $k_{s}$ and corresponding reductions in $\mathcal{R}_{0}$, one has to test the entire undetected population once every $N$ days (or equivalently every day a random fraction of $1 / N)$. To improve the efficiency of testing, i.e., the probability per test of getting a positive result by avoiding unnecessary testing of people who have a low likelihood of being infected, one can reduce the sample population by the following approaches:

1. Serological testing: With serological testing one can remove the recovered population from the pool of undetected individuals, and thus the same number of positive test results can be achieved with fewer tests. However, during an early stage of the pandemic the relative size of the undetected recovered compared to the whole undetected population is very small, and therefore the gain would be negligible. Nevertheless, this approach can easily be integrated into any mass testing strategy once reliable serological tests become available. Here, we can assume that immune individuals will remain immune for an extended period of time (e.g. up to 1-2 years; however, this 
is still subject to verification). If immune people cannot be infected for a second time (or are infected at a much lower rate than susceptible individuals), one can collect the information on positive test results to exclude the immune individuals from the Covid-19 RNA testing.

2. Inference from contact tracing: A more effective approach would be based on an inference model (e.g. by using contact tracing of infected individuals), which allows to divide the sample population $\mathcal{D}$ into one subpopulation $\tilde{\mathcal{D}}$ with a higher and the remainder with a lower percentage of infected individuals. For the following analysis we denote the size of $\mathcal{D}$ with $n$ and that of $\tilde{\mathcal{D}}$ with $\tilde{n}$. Further, $\tilde{p}$ is the fraction of infected persons in $\tilde{\mathcal{D}}$ and $p$ that in $\mathcal{D}$. Testing every person in $\tilde{\mathcal{D}}$ at a frequency of $1 / N$ would require $\tilde{n} / N$ tests per day; opposed to $n / N$ tests, if the whole sample population was tested. The respective numbers of positive test results per day, on the other hand, would be $\tilde{P}(N)=\tilde{p} \tilde{n} / N$ opposed to $P(N)=p n / N$. In order to obtain the same number of positive results from the subpopulation $\tilde{\mathcal{D}}$ as one would get from $\mathcal{D}$, one has to reduce the test interval $N$ to $\tilde{N}$, such that $\tilde{P}(\tilde{N})=P(N)$. From this one obtains $\tilde{N}=N(\tilde{p} \tilde{n}) /(p n)$ and one can conclude that the number of tests required to achieve the same overall quota reduces by the factor

$$
r=\frac{\tilde{n} / \tilde{N}}{n / N}=\frac{N \tilde{n}}{\tilde{N} n}=\frac{p}{\tilde{p}} .
$$

In order for this result to be practically meaningful, $\tilde{N}$ has to be at least one, which translates into the requirement that

$$
\frac{\tilde{n}}{n} \geq \frac{r}{N}
$$

In short, if one can identify a subpopulation $\tilde{\mathcal{D}} \subset \mathcal{D}$ for which the percentage of infections is higher by a factor of $r^{-1}$ than in $\mathcal{D}$, and which is larger than $n r / N$, then the number of tests needed to obtain the same reproduction number reduces by the factor $r$. The curves in Fig. 3B show the relationship between number of tests per 100'000 people per day needed to achieve $\mathcal{R}_{0}=1$ and the prevalence ratio between sub- and overall population; in combination with mild social distancing (solid line) and without social distancing (dashed line).

\section{Contact Counting - How to Screen Large Enough Subpopulations with High Prevalence}

Here we devise a way to screen large enough subpopulations with a much higher prevalence and infectiousness than the overall population, which is a prerequisite for smart testing.

We study three subpopulations as potential candidates for our smart testing mitigation approach; of interest are their prevalence, their infectiousness and their size. Next we describe them and provide quantitative estimates for the most relevant subpopulation.

1. Contacts of symptomatic cases: A straight-forward approach would be to choose the contacts of symptomatic cases as our subpopulation. While this group is highly likely to be infected, this approach has one major drawback. In fact, the outcome of testing contacts of symptomatic cases would not be much different than that of contact tracing mentioned before (and discussed in detail by Ferretti et al.[7]). Therefore, it suffers from the same limitation of not catching sufficient numbers of asymptomatic infections. Besides tracing contacts which potentially got infected by a symptomatic individual, one may also find the contact by whom it got infected. That person has most likely recovered, since he/she got infected roughly 10-14 days ago. Therefore this contact would not be tested positive (as virus titers may already be low and as we still lack reliable serological tests) and hence testing contacts of symptomatic ones would not lead us to a larger group with a sufficient number of asymptomatic cases. 
2. Direct and indirect contacts of symptomatic cases: One way to cope with the issue arising from lack of enough asymptomatic cases in the contacts of symptomatic ones is to enlarge our sample population and include also indirect contacts of symptomatic individuals in the past two weeks. This strategy, while most probably catching enough asymptomatic cases, may not reduce the burden of mass testing, since now the size of the subpopulation becomes simply too large. This problem has also been noted by others [10]. For example at a prevalence of $1 \%$ in the total population, and assuming 10 contacts per person, the size of this subpopulation becomes almost as large as the whole population.

3. High-contact individuals (Fig. 4A): In this scenario we only test those with significantly more contacts than the average. In the following we show that indeed this strategy allows to screen a high prevalence subpopulation which is also large enough to stop the pandemic. It is also important to emphasize that the prerequisite of this strategy is to utilize a contact counter, which may be integrated into an existing contact tracing app that uses bluetooth technology.

While the improvements resulting from contact counting can be estimated based on the prevalence ratio, the contact counting scheme has a more fundamental feature that exhibits itself directly in the basic reproduction number. In fact by cutting out the highly transmissive parts of the population network, we reduce the basic reproduction number significantly. This reduction in $\mathcal{R}_{0}$ can be evaluated by considering the transmissibility of the disease $T$. In short, considering a normalized recovery rate, $T$ is the probability that an infected person infects one of their contacts per unit of time. Consider $\mathcal{K}$ to be the degree of connectivity of a person; therefore we can compute $T$ for a heterogeneous network as $[19,22]$

$$
T=\mathcal{R}_{0} \frac{\mathbb{E}[\mathcal{K}]}{\mathbb{E}\left[\mathcal{K}^{2}-\mathcal{K}\right]}
$$

Now imagine a scenario where we halt the virus-spread among all individuals with a degree of connectivity above $\mathcal{K}_{0}$; for example via vaccinating every person who has contact numbers above $\mathcal{K}_{0}$. Therefore, we get a reduction in the virus reproduction number

$$
\mathcal{R}_{0}^{n n}=\frac{\mathbb{E}\left[\mathcal{K}^{2}-\mathcal{K} \mid \mathcal{K} \leq \mathcal{K}_{0}\right]}{\mathbb{E}\left[\mathcal{K}^{2}-\mathcal{K}\right]} \frac{\mathbb{E}[\mathcal{K}]}{\mathbb{E}\left[\mathcal{K} \mid \mathcal{K} \leq \mathcal{K}_{0}\right]} \mathcal{R}_{0},
$$

where $\mathcal{R}_{0}^{n n}$ denotes the basic reproduction number of the new network. For a specified size of the new network this would be the maximum reduction one could achieve by reshaping the network, as the tail of the contact distribution is removed (Fig. 4B). However, in practice there are failures in containing the virus-spread through highly connected people. The efficacy $\mathcal{E}$ of a smart testing based on contact counting thus depends on the number of highly connected people who would employ the contact counting app, as well as the accuracy of the tests. In the following we compute how these boundary conditions affect STeCC. However, before proceeding, notice that we suppose that the isolated individuals have negligible contributions to the virus-spread. Furthermore, we assume that elderly people (above 70 years of age) are shielded by isolation and that children below 10 years would not contribute to the infection dynamics $[26,9]$. Therefore our target subpopulation is considered to be in possession of smart-phones.

Let us define a testing regime, where we screen through app users with number of connections $\mathcal{K} \geq \mathcal{K}_{0}^{(1)}$ at day $1, \mathcal{K}_{0}^{(2)} \leq \mathcal{K}_{0} \leq \mathcal{K}_{0}^{(1)}$ at day 2 and so-forth until testing $\mathcal{K}_{0}^{\left(T_{t}\right)} \leq \mathcal{K}_{0} \leq \mathcal{K}_{0}^{\left(T_{t}-1\right)}$ at day $T_{t}$. Consequently, based on the test results, we ask the positively tested individuals to quarantine themselves. We fix the testing cycle to $T_{t}=7$ days (see Fig. 4). Consider $\zeta$ to be the fraction of smart-phone owners who utilize the app. The portion of the network besides the fraction $\zeta$ that we can disconnect from the population depends on the probability of the event that a positively tested highly connected individual could pass on the virus to at least one person in the past $T_{t}+\tau_{\text {proc }}$ days. 
Let us denote such an event by $\mathcal{A}_{\text {out }}$ and consider the test processing time $\tau_{\text {proc }}$. Therefore the reduction in $\mathcal{R}_{0}$ during the whole cycle of STeCC becomes

$$
\mathcal{R}_{0}^{n n}=\frac{\mathcal{P}_{\mathcal{K} \leq \mathcal{K}_{0}^{\left(T_{t}\right)}} \mathbb{E}\left[\mathcal{K}^{2}-\mathcal{K} \mid \mathcal{K} \leq \mathcal{K}_{0}^{\left(T_{t}\right)}\right]+(1-\mathcal{E}) \mathcal{P}_{\mathcal{K} \geq \mathcal{K}_{0}^{\left(T_{t}\right)}} \mathbb{E}\left[\mathcal{K}^{2}-\mathcal{K} \mid \mathcal{K} \geq \mathcal{K}_{0}^{\left(T_{t}\right)}\right]}{\mathcal{P}_{\mathcal{K} \leq \mathcal{K}_{0}^{\left(T_{t}\right)}} \mathbb{E}\left[\mathcal{K} \mid \mathcal{K} \leq \mathcal{K}_{0}^{\left(T_{t}\right)}\right]+(1-\mathcal{E}) \mathcal{P}_{\mathcal{K} \geq \mathcal{K}_{0}^{\left(T_{t}\right)}} \mathbb{E}\left[\mathcal{K} \mid \mathcal{K} \geq \mathcal{K}_{0}^{\left(T_{t}\right)}\right]} \frac{\mathbb{E}[\mathcal{K}]}{\mathbb{E}\left[\mathcal{K}^{2}-\mathcal{K}\right]} \mathcal{R}_{0},
$$

where $\mathcal{E}$ is the efficacy of STeCC and can be computed via

$$
\mathcal{E}=1-\left(\left(\mathcal{P}_{\mathcal{A}_{\text {out }}}(1-\eta)+\eta\right) \zeta+(1-\zeta)\right) .
$$

Note that $\eta$ is the fraction of false negative test results, $\mathcal{P}_{\mathcal{A}_{\text {out }}}$ is the probability of the event $\mathcal{A}_{\text {out }}, \mathcal{P}_{\mathcal{K} \leq \mathcal{K}_{0}^{\left(T_{t}\right)}}$ is the probability that an individual has contacts below $\mathcal{K}_{0}^{\left(T_{t}\right)}$ and $\mathcal{P}_{\mathcal{K} \geq \mathcal{K}_{0}^{\left(T_{t}\right)}}=1-\mathcal{P}_{\mathcal{K} \leq \mathcal{K}_{0}^{\left(T_{t}\right)}}$. It is evident that $\mathcal{R}_{0}^{n n}$ and $\mathcal{E}$ would now depend on the network topology and latency time, respectively.

\subsection{Network Topology}

In order to model the heterogeneity of a population relevant for disease modeling, scale-free networks offer appropriate features $[5,19,16,22]$. Scale-free networks are characterized by a power-law distribution which determines the probability density function $P_{k}$ of the degree of connectivity $k$ per node. We employ a continuous approximation [23], that is,

$$
P_{k}=\alpha_{p} k^{-(2+\gamma)} \quad\left(m \leq k \leq k_{c}\right) \& \quad(0 \leq \gamma \leq 1)
$$

where

$$
\alpha_{p}=\frac{(1+\gamma) m^{1+\gamma}}{1-\left(k_{c} / m\right)^{-(1+\gamma)}} .
$$

Notice that by contacts we mean disease relevant contacts. We adopt $\gamma=0.3$ and fix the upper and lower cut-offs by $k_{c}=552$ and $m=4$, respectively. These choices were made in order to have a realistic range for number of contacts and obtain an average number of contacts of $\mu=13.4$, which is consistent with the data provided in [21]. Two extreme choices of $\gamma$ include $\gamma=1$ and $\gamma=0$. While the former leads to the celebrated Barabási and Albert (BA) model [1]; the latter has been used in disease spread models, e.g. see [19]. Note that we conducted a sensitivity analysis of our results with respect to $\gamma$, see Figs. S7 and S8.

With the choice of the degree of connectivity distribution we can compute the relative size of the population with a degree of connectivity above a certain $\mathcal{K}_{0}^{\left(T_{t}\right)}$ :

$$
\mathcal{P}_{\mathcal{K} \geq \mathcal{K}_{0}^{\left(T_{T}\right)}}=\frac{\alpha_{p}}{1+\gamma}\left(\mathcal{K}_{0}^{\left(T_{t}\right)^{-(1+\gamma)}}-k_{c}^{-(1+\gamma)}\right) .
$$

Before proceeding further, let us mention that for the perfect efficacy $\mathcal{E}=1$, we can reach $\mathcal{R}_{0}^{n n}=1$ with $\mathcal{K}_{0}^{\left(T_{t}\right)}=138.8$. This accounts for $0.83 \%$ of the population with highest degree of connectivity. Now, to translate $\mathcal{P}_{\mathcal{K} \geq \mathcal{K}_{0}^{\left(T_{t}\right)}}$ of the population into the equivalent number of tests, suppose we conduct $N_{\text {test }}$ per day per 100 '000 people. Thus we obtain

$$
\mathcal{K}_{0}^{\left(T_{t}\right)}=\left(\frac{(1+\gamma) N_{\text {test }} T_{t}}{10^{5} \alpha_{p} \zeta}+k_{c}^{-(1+\gamma)}\right)^{-1 /(\gamma+1)} .
$$




\subsection{Uncontained Virus Spread}

Since we test these high-contact individuals once every $T_{t}=7$ days (test cycle depicted in Fig. 4B), there is a chance that a virus-positive person has already transmitted the virus between two successive tests. The probability of such events depends on the latency time $\tau_{l}$ and average number of contacts of the individual. The infection time $\tau_{0}$ is uniformly distributed between 0 and $T_{t}$. Now, since these individuals have contacts way above average, we take a conservative estimation that

$$
\mathcal{P}_{\mathcal{A}_{\text {out }}}=\operatorname{Prob}\left\{\left(\tau_{0}+\tau_{1}\right) \leq\left(T_{t}+\tau_{\text {proc }}\right)\right\}
$$

which becomes 0.4296 for the case of $T_{t}=7$ (day) and $\tau_{\text {proc }}=1$ (day).

\subsection{Scenario Analysis}

Now we are ready to consider the following scenarios and compute the reduction of the basic reproduction number as a function of number of app users.

1. Scneario A: In this STeCC alone scenario we identify high-contact individuals in cycles of 7 days (Fig. 4B), ask them to be tested and ask the positively tested individuals to go into self-quarantine. We compute the reduced basic reproduction number $\mathcal{R}_{0}^{S T-A}$ using Eq. (37) with the efficacy equation (38). For our base parameters together with the network topology with $\gamma=0.3, m=4$ and $k_{c}=552$, Fig. S 6 shows the performance of STeCC-A. While up to $30 \%$ reduction of $\mathcal{R}_{0}$ can be achieved with a combination of $90 \%$ app users among smart phone users (which would only be achieved in very optimistic scenarios) and 200 tests per 100 '000 per day, it is clearly insufficient to halt the pandemic. The reason lies in the fact that we would not be able to contain the virus spread from almost $40 \%$ of highly connected people resulting from the transmission events that occur between their infection and the date of the virus test. Next, we introduce a combination that can significantly reduce this virus spread.

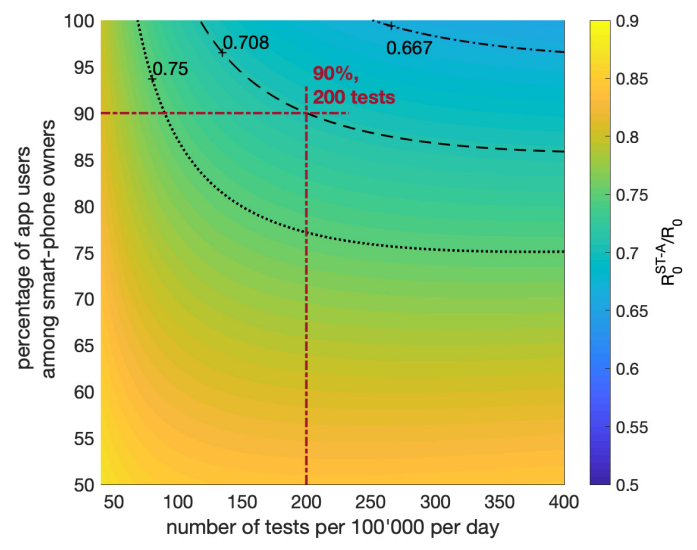

Fig. S 6. $\mathrm{STeCC}$ alone scenario (STeCC-A): $\mathcal{R}_{0}^{S T-A} / \mathcal{R}_{0}$ as function of number of smart tests per $100^{\prime} 000$ per day and the percentage of smartphone owners who participate in STeCC (Scenario A). For $\mathcal{R}_{0}=2.4$, the dotted line indicates $\mathcal{R}_{0}^{S T-A}=1.8$, the dashed line $\mathcal{R}_{0}^{S T-A}=1.7$ and the dashed-dotted line $\mathcal{R}_{0}^{S T-A}=1.6$. 
2. Scenario B: Here we consider a variant of $\mathrm{STeCC}$ in which besides the positively tested ones also their contacts are asked to quarantine. This results in a much lower basic reproduction number of $\mathcal{R}_{0}^{S T-B}$, which is obtained from Eq. (37) with the improved efficacy

$$
\mathcal{E}=\mathcal{E}^{S T-B}=1-\left((\mathcal{P}_{\mathcal{A}_{\text {out }}}(1-\eta) \overbrace{(1-\zeta)}^{\text {due to quarantine }}+\eta) \zeta+(1-\zeta)\right) .
$$

The results shown in Fig. 4C predict a much stronger effect than STeCC alone (Fig. S 6). The combination of $90 \%$ user percentage and almost 400 tests per $100 ' 000$ per day now lead to $\mathcal{R}_{0}^{S T-B}=1$. Again, this approach alone will likely not suffice to halt the pandemic under realistic conditions.

3. Scenario $C$ : Since the app already provides the contacts, we can further improve the STeCC based mitigation by combining it with conventional contact tracing (see $\$ 4$ ). This is especially interesting since neither contact tracing nor STeCC alone are effective enough to halt the pandemic under realistic conditions. Therefore, on top of scenario B, we also ask the contacts of symptomatic cases to quarantine, which leads to a basic reproduction number of

$$
\mathcal{R}_{0}^{S T-C}=\frac{\mathcal{P}_{\mathcal{K} \leq \mathcal{K}_{0}^{\left(T_{t}\right)}} \mathbb{E}\left[\mathcal{K}^{2}-\mathcal{K} \mid \mathcal{K} \leq \mathcal{K}_{0}^{\left(T_{t}\right)}\right]+\left(1-\mathcal{E}^{S T-B}\right) \mathcal{P}_{\mathcal{K} \geq \mathcal{K}_{0}^{\left(T_{t}\right)}} \mathbb{E}\left[\mathcal{K}^{2}-\mathcal{K} \mid \mathcal{K} \geq \mathcal{K}_{0}^{\left(T_{t}\right)}\right]}{\mathcal{P}_{\mathcal{K} \leq \mathcal{K}_{0}^{\left(T_{t}\right)}} \mathbb{E}\left[\mathcal{K} \mid \mathcal{K} \leq \mathcal{K}_{0}^{\left(T_{t}\right)}\right]+\left(1-\mathcal{E}^{S T-B}\right) \mathcal{P}_{\mathcal{K} \geq \mathcal{K}_{0}^{\left(T_{t}\right)}} \mathbb{E}\left[\mathcal{K} \mid \mathcal{K} \geq \mathcal{K}_{0}^{\left(T_{t}\right)}\right]} \frac{\mathbb{E}[\mathcal{K}]}{\mathbb{E}\left[\mathcal{K}^{2}-\mathcal{K}\right]} \mathcal{R}_{0}^{c t},
$$

where $\mathcal{R}_{0}^{c t}=(1-\zeta \kappa) \mathcal{R}_{0}$ and $\kappa=\mathcal{R}_{0}^{\text {sym }} / \mathcal{R}_{0}$ (see $\S 4$ ). The result corresponding to this scenario is shown in Fig. 4D. Accordingly, we predict that $\mathcal{R}_{0}^{S T-C}=1$ can be achieved with $72 \%$ app users among smart phone users and 166 tests per 100'000 per day. This is very encouraging, since 72\% app users among smart phone users corresponds to only about 50\% app users of the whole population. Furthermore, a testing capacity of 166 per 100'000 per day already is available in several developed countries, including Switzerland.

\subsection{Sensitivity Study}

In order to gain further confidence in our STeCC related mitigation scenarios, we conducted studies to investigate the sensitivity of the basic reproduction number $\mathcal{R}_{0} \in\{1.9,2.9,3.4\}$. The parameters which we varied are $\gamma \in\{2,2.5,3.5\}$ in the exponent of the power-law distribution of the degree of connectivity, the ratio $\eta \in\{0.1,0.15\}$ of false negatives and the test processing time $\tau_{\text {proc }} \in\{0.5,1.5\}$ (day). Note that our base setting is the combination of $\mathcal{R}_{0}=2.4, \gamma=0.3$, $\eta=0.05$ and $\tau_{\text {proc }}=1$ (day). Figures S 7 and 8 show the sensitivity of STeCC-B and-C scenarios, respectively, for varying $\mathcal{R}_{0}$ and $\gamma$ values. Figure S9 depicts the sensitivity of STeCC-C with respect to the fraction of false negatives and test processing time. We observe that for a large range of parameters considered the combination of STeCC and conventional contact tracing leads to stopping the pandemic with realistic app user percentage (i.e., $60 \%$ to $85 \%$ ) and number of tests per day (i.e., 50 to 350 tests per 100'000).

\section{Model Implementation}

The dynamic model was implemented with Maple 2018. The calculations for mass testing, contact tracing and smart testing were implemented with MATLAB and the Statistics Toolbox Release 2018b. 


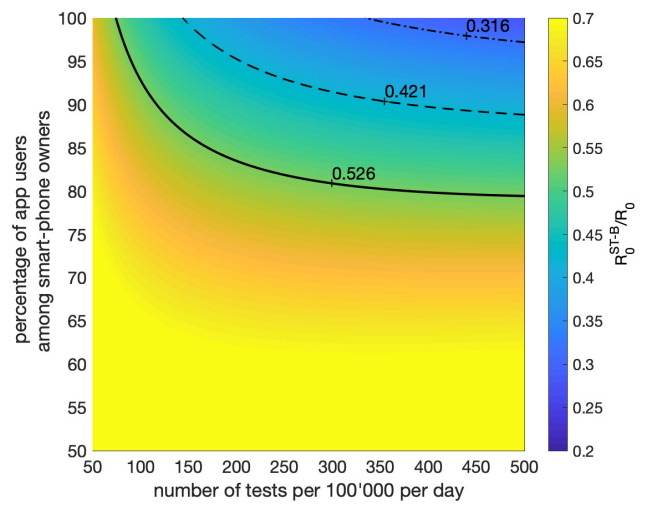

(A)

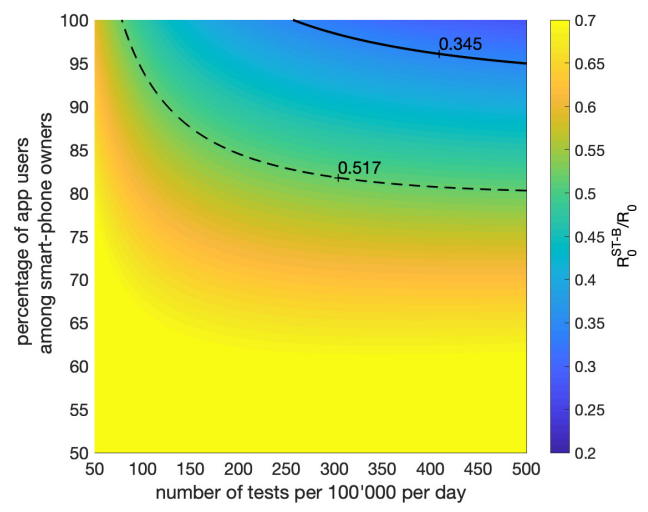

(C)

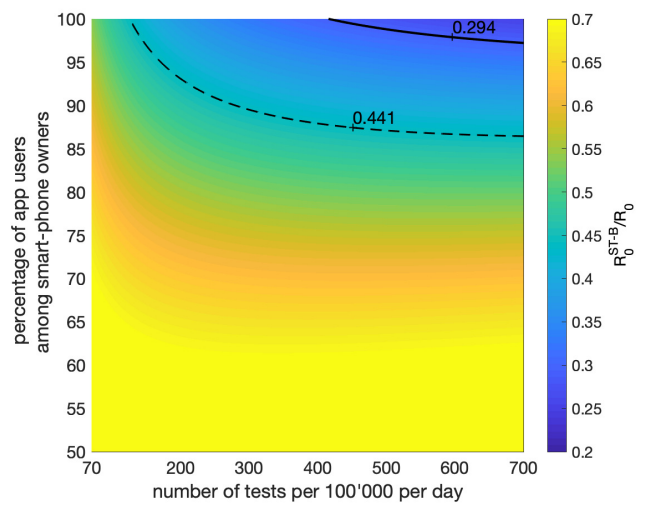

(E)

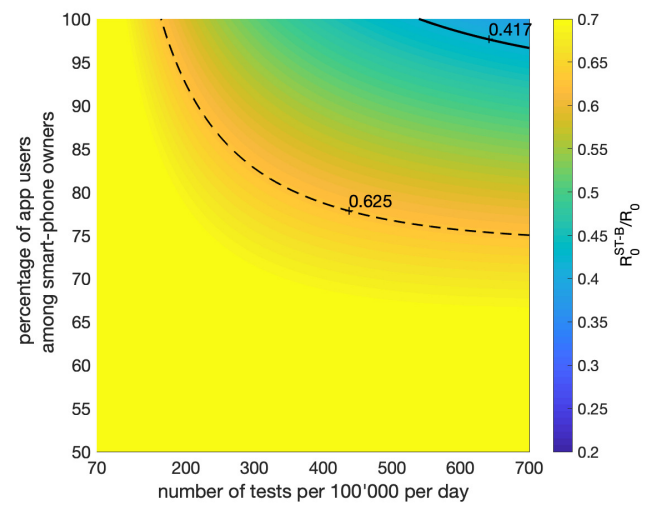

(B)

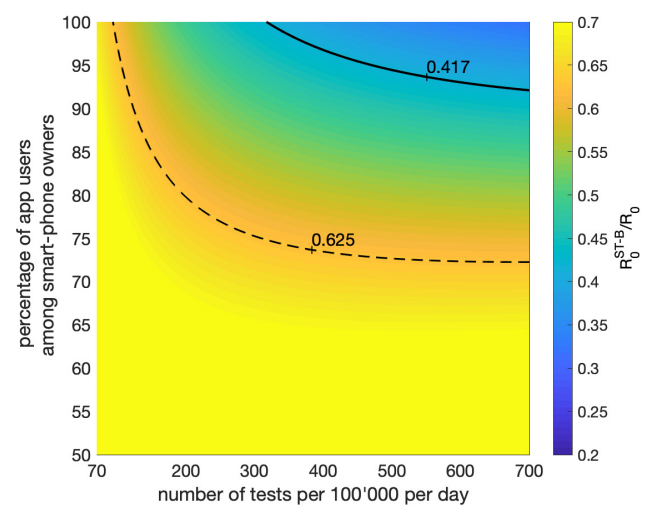

(D)

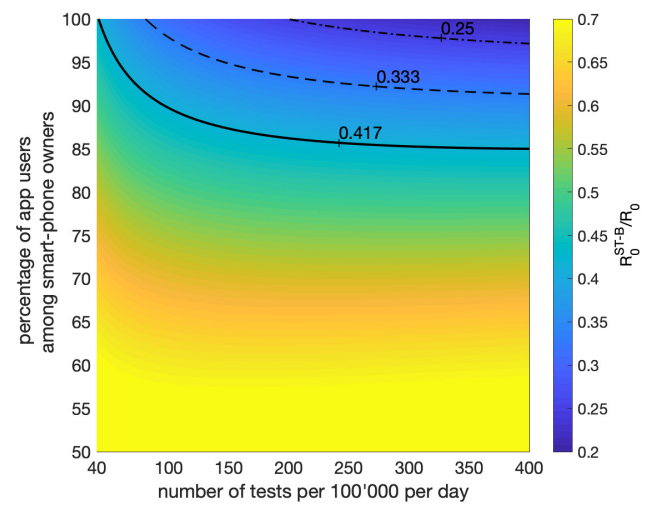

(F)

Fig. S 7. STeCC plus isolation of contacts with positively tested individuals (STeCC-B): $\mathcal{R}_{0}^{S T-B} / \mathcal{R}_{0}$ as function of number of smart tests per $100^{\prime} 000$ per day and the percentage of smart-phone owners who participate in STeCC (scenario B). For $\left(\gamma=0.3, k_{c}=552\right)$ and $(\mathrm{A}) \mathcal{R}_{0}=1.9$, (C) $\mathcal{R}_{0}=2.9$ and (E) $\mathcal{R}_{0}=3.4$; for $\mathcal{R}_{0}=2.4$ and (B) $\left(\gamma=0.2, k_{c}=227\right)$, (D) $\left(\gamma=0.25, k_{c}=327\right)$ and (F) $\left(\gamma=0.35, k_{c}=1325\right)$. A corresponding map with $\mathcal{R}_{0}=2.4$ and $\gamma=0.3$ is shown in Fig. 4C. The bold lines indicates the combinations for which $\mathcal{R}_{0}^{S T-B}=1$. 


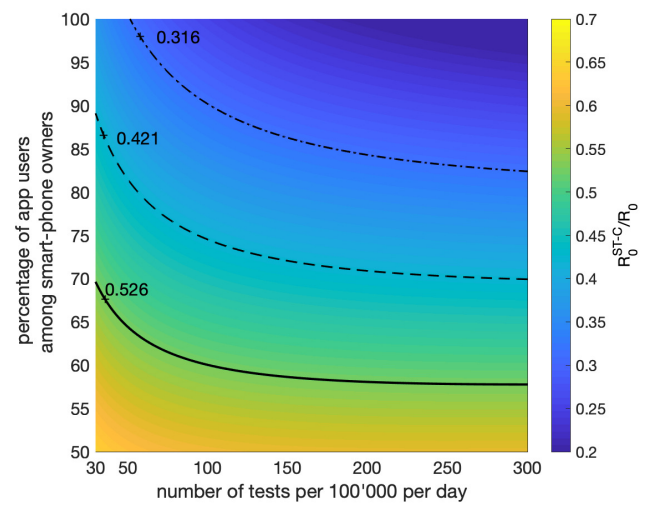

(A)

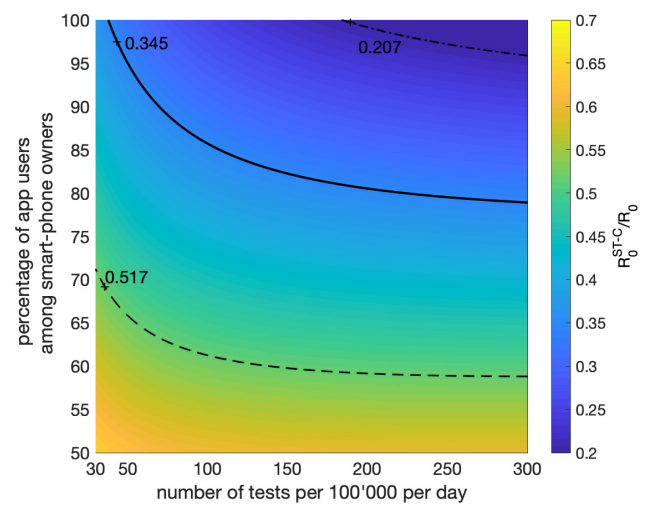

(C)

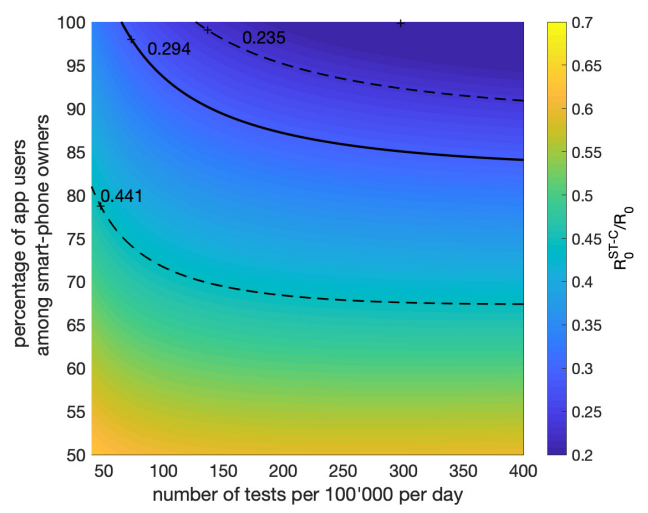

(E)

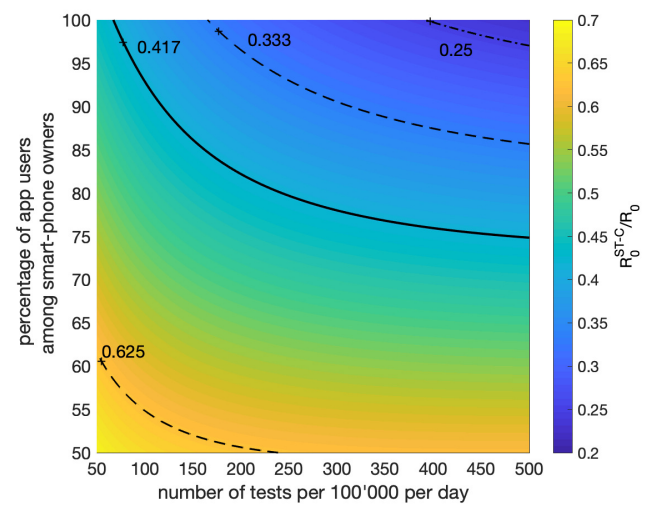

(B)

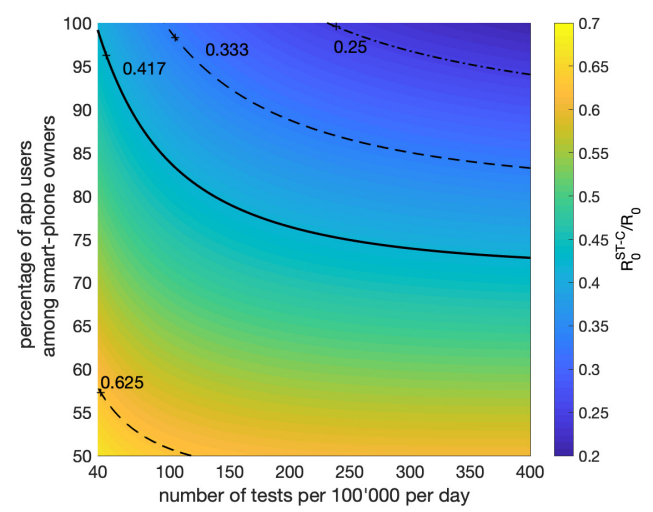

(D)

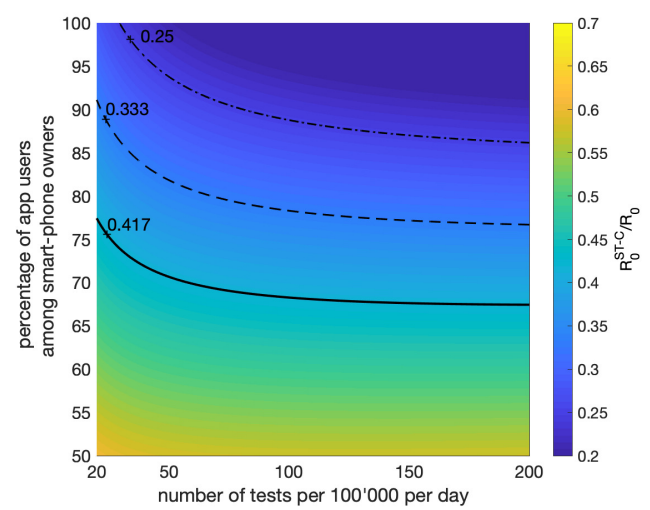

(F)

Fig. S 8. STeCC plus isolation of contacts with positively tested individuals plus classical contact tracing (STeCC-C): $\mathcal{R}_{0}^{S T-C} / \mathcal{R}_{0}$ as function of number of smart tests per $100^{\prime} 000$ per day and the percentage of smart-phone owners who participate in STeCC (scenario C). For $\left(\gamma=0.3, k_{c}=552\right)$ and (A) $\mathcal{R}_{0}=1.9$, (C) $\mathcal{R}_{0}=2.9$ and (E) $\mathcal{R}_{0}=3.4$; for $\mathcal{R}_{0}=2.4$ and (B) $\left(\gamma=0.2, k_{c}=227\right)$, (D) $\left(\gamma=0.25, k_{c}=327\right)$ and (F) $\left(\gamma=0.35, k_{c}=1325\right)$. A corresponding map with $\mathcal{R}_{0}=2.4$ and $\gamma=0.3$ is shown in Fig. 4D. The bold lines indicates the combinations for which $\mathcal{R}_{0}^{S T-C}=1$. 


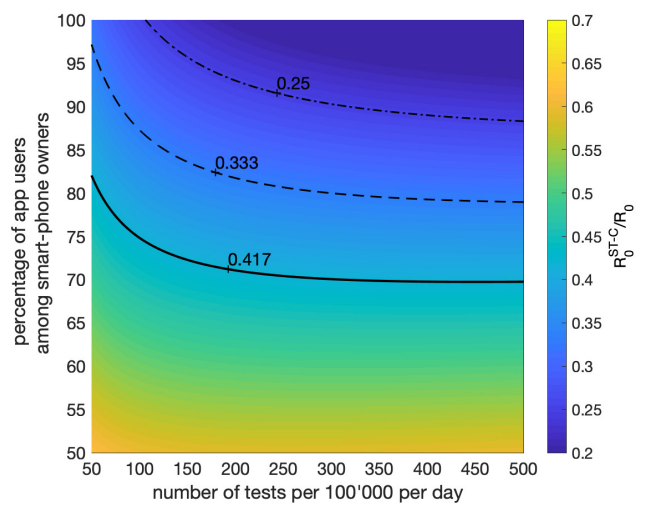

(A)

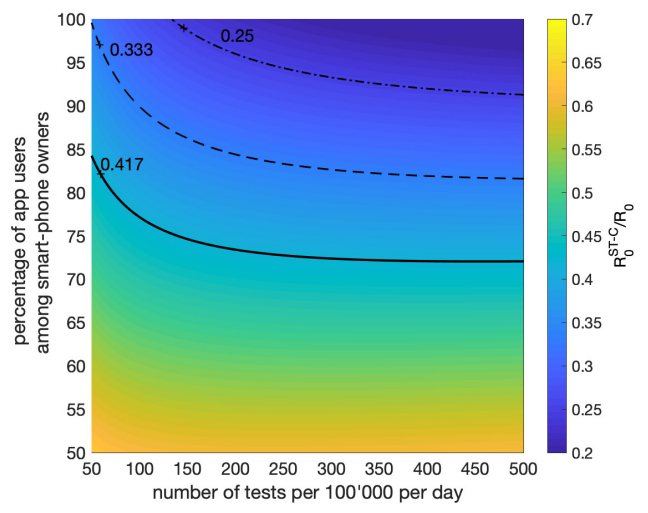

(C)

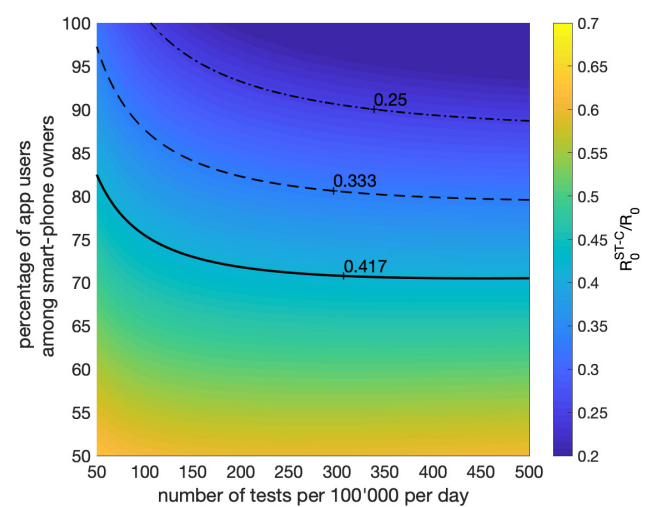

(B)

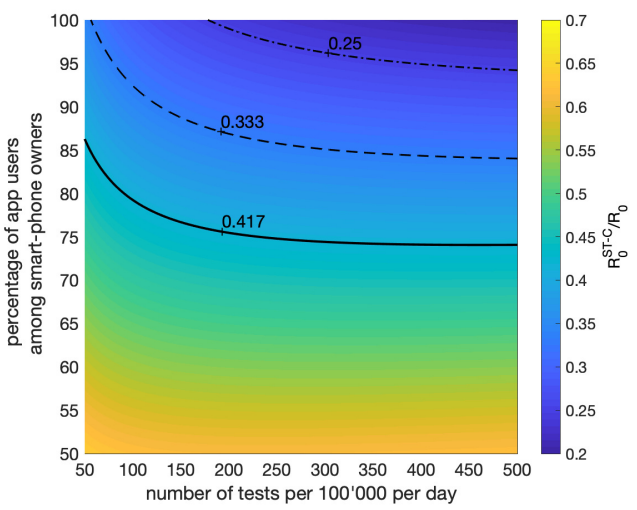

(D)

Fig. S 9. STeCC plus isolation of contacts with positively tested individuals plus classical contact tracing (STeCC-C): $\mathcal{R}_{0}^{S T-C} / \mathcal{R}_{0}$ as function of number of smart tests per $100^{\prime} 000$ per day and the percentage of smart-phone owners who participate in STeCC (scenario C). For $(\eta=0.05)$ and (A) $\tau_{\text {proc }}=0.5$ (day) and (C) $\tau_{\text {proc }}=1.5$ (day); for $\tau_{\text {proc }}=1$ (day) and (B) $\eta=0.1$, (D) $\eta=0.15$. A corresponding map with $\eta=0.05$ and $\tau_{\text {proc }}=1$ (day) is shown in Fig. 4D. The bold lines indicates the combinations for which $\mathcal{R}_{0}^{S T-C}=1$. 


\section{References}

[1] Albert-László Barabási and Réka Albert. Emergence of scaling in random networks. science, 286(5439):509-512, 1999.

[2] David Baud, Xiaolong Qi, Karin Nielsen-Saines, Didier Musso, Léo Pomar, and Guillaume Favre. Real estimates of mortality following covid-19 infection. The Lancet Infectious Diseases, 2020.

[3] Victor M Corman, Olfert Landt, Marco Kaiser, Richard Molenkamp, Adam Meijer, Daniel KW Chu, Tobias Bleicker, Sebastian Brünink, Julia Schneider, Marie Luisa Schmidt, Daphne GJC Mulders, Bart L Haagmans, Bas van der Veer, Sharon van den Brink, Lisa Wijsman, Gabriel Goderski, Jean-Louis Romette, Joanna Ellis, Maria Zambon, Malik Peiris, Herman Goossens, Chantal Reusken, Marion PG Koopmans, and Christian Drosten. Detection of 2019 novel coronavirus (2019-nCoV) by real-time RT-PCR. Eurosurveillance, 25(3), January 2020. doi: 10.2807/1560-7917.es.2020.25.3.2000045. URL https ://doi.org/10.2807/1560-7917.es . 2020. 25. 3.2000045

[4] Odo Diekmann, Johan Andre Peter Heesterbeek, and Johan AJ Metz. On the definition and the computation of the basic reproduction ratio $r_{0}$ in models for infectious diseases in heterogeneous populations. Journal of mathematical biology, 28(4):365-382, 1990.

[5] Victor M Eguiluz and Konstantin Klemm. Epidemic threshold in structured scale-free networks. Physical Review Letters, 89(10):108701, 2002.

[6] Neil M Ferguson, Daniel Laydon, Gemma Nedjati-Gilani, Natsuko Imai, Kylie Ainslie, Marc Baguelin, Sangeeta Bhatia, Adhiratha Boonyasiri, Zulma Cucunubá, Gina Cuomo-Dannenburg, et al. Impact of non-pharmaceutical interventions (npis) to reduce covid-19 mortality and healthcare demand. London: Imperial College COVID-19 Response Team, March, 16, 2020.

[7] Luca Ferretti, Chris Wymant, Michelle Kendall, Lele Zhao, Anel Nurtay, Lucie Abeler-Dörner, Michael Parker, David Bonsall, and Christophe Fraser. Quantifying sars-cov-2 transmission suggests epidemic control with digital contact tracing. Science, 2020.

[8] Christophe Fraser, Steven Riley, Roy M Anderson, and Neil M Ferguson. Factors that make an infectious disease outbreak controllable. Proceedings of the National Academy of Sciences, 101(16):6146-6151, 2004.

[9] Daniel F Gudbjartsson, Agnar Helgason, Hakon Jonsson, Olafur T Magnusson, Pall Melsted, Gudmundur L Norddahl, Jona Saemundsdottir, Asgeir Sigurdsson, Patrick Sulem, Arna B Agustsdottir, et al. Spread of sarscov-2 in the icelandic population. New England Journal of Medicine, 2020.

[10] Robert Hinch, Will Probert, Anel Nurtay, Michelle Kendall, Chris Wymant, Matthew Hall, Katrina Lythgoe, Ana Bulas Cruz, Lele Zhao, Andrea Stewart, Luca Ferretti, Michael Parker, Ares Meroueh, Bryn Mathias, Scott Stevenson, Daniel Montero, James Warren, Nicole K Mather, Anthony Finkelstein, Lucie, Abeler-Dörner, David Bonsall, and Christophe Fraser. Effective configurations of a digital contact tracing app: A report to NHSX, April 2020. URL https://045.medsci.ox.ac.uk/files/files/report-effective-app-configurations . pdf.

[11] Ayaan Hossain, C. Alexander Reis, Sarthok Rahman, and M. Howard Salis. A massively parallel covid-19 diagnostic assay for simultaneous testing of 19200 patient samples, 2020. 
[12] Enrico Lavezzo, Elisa Franchin, Constanze Ciavarella, Gina Cuomo-Dannenburg, Luisa Barzon, Claudia Del Vecchio, Lucia Rossi, Riccardo Manganelli, Arianna Loregian, Nicolò Navarin, et al. Suppression of covid-19 outbreak in the municipality of vo, italy. medRxiv, 2020.

[13] Ruiyun Li, Sen Pei, Bin Chen, Yimeng Song, Tao Zhang, Wan Yang, and Jeffrey Shaman. Substantial undocumented infection facilitates the rapid dissemination of novel coronavirus (sars-cov2). Science, 2020.

[14] Ying Liu, Albert A Gayle, Annelies Wilder-Smith, and Joacim Rocklöv. The reproductive number of covid-19 is higher compared to sars coronavirus. Journal of travel medicine, 2020.

[15] Zhonghua Liu, Xing Bing, and Xue Za Zhi. The epidemiological characteristics of an outbreak of 2019 novel coronavirus diseases (covid-19) in china. Novel Coronavirus Pneumonia Emergency Response Epidemiology Team, 41(2):145, 2020.

[16] Robert M May and Alun L Lloyd. Infection dynamics on scale-free networks. Physical Review E, 64(6):066112, 2001.

[17] Kenneth McIntosh, Martin S Hirsch, and Allyson Bloom. Coronavirus disease 2019 (covid-19). UpToDate, 5, 2020.

[18] Ke Men, Xia Wang, Yihao Li, Guangwei Zhang, Jingjing Hu, Yanyan Gao, and Henry Han. Estimate the incubation period of coronavirus 2019 (covid-19). medRxiv, 2020.

[19] Lauren Ancel Meyers, Babak Pourbohloul, Mark EJ Newman, Danuta M Skowronski, and Robert C Brunham. Network theory and sars: predicting outbreak diversity. Journal of theoretical biology, 232(1):71-81, 2005.

[20] Kenji Mizumoto and Gerardo Chowell. Estimating risk for death from 2019 novel coronavirus disease, china, january-february 2020. Emerging infectious diseases, 26(6), 2020.

[21] Joël Mossong, Niel Hens, Mark Jit, Philippe Beutels, Kari Auranen, Rafael Mikolajczyk, Marco Massari, Stefania Salmaso, Gianpaolo Scalia Tomba, Jacco Wallinga, et al. Social contacts and mixing patterns relevant to the spread of infectious diseases. PLoS medicine, 5(3), 2008.

[22] Mark EJ Newman. Spread of epidemic disease on networks. Physical review E, 66(1):016128, 2002.

[23] Romualdo Pastor-Satorras and Alessandro Vespignani. Epidemic dynamics in finite size scale-free networks. Physical Review E, 65(3):035108, 2002.

[24] Robert Verity, Lucy C Okell, Ilaria Dorigatti, Peter Winskill, Charles Whittaker, Natsuko Imai, Gina CuomoDannenburg, Hayley Thompson, Patrick GT Walker, Han Fu, et al. Estimates of the severity of coronavirus disease 2019: a model-based analysis. The Lancet Infectious Diseases, 2020.

[25] Victor Virlogeux, Vicky J Fang, Joseph T Wu, Lai-Ming Ho, JS Malik Peiris, Gabriel M Leung, and Benjamin J Cowling. Incubation period duration and severity of clinical disease following severe acute respiratory syndrome coronavirus infection. Epidemiology (Cambridge, Mass.), 26(5):666, 2015.

[26] Yi Xu, Xufang Li, Bing Zhu, Huiying Liang, Chunxiao Fang, Yu Gong, Qiaozhi Guo, Xin Sun, Danyang Zhao, Jun Shen, et al. Characteristics of pediatric sars-cov-2 infection and potential evidence for persistent fecal viral shedding. Nature medicine, pages 1-4, 2020.

[27] Fei Zhou, Ting Yu, Ronghui Du, Guohui Fan, Ying Liu, Zhibo Liu, Jie Xiang, Yeming Wang, Bin Song, Xiaoying $\mathrm{Gu}$, et al. Clinical course and risk factors for mortality of adult inpatients with covid-19 in wuhan, china: a retrospective cohort study. The Lancet, 2020. 